\title{
Utilitarian mechanism design for an excludable public good
}

\author{
Martin F. Hellwig
}

Received: 15 January 2009 / Accepted: 25 June 2009 / Published online: 14 July 2009 (C) The Author(s) 2009. This article is published with open access at Springerlink.com

\begin{abstract}
This paper studies the design of optimal utilitarian mechanisms for an excludable public good. Excludability provides a basis for making people pay for admissions; the payments can be used for redistribution and/or funding. Whereas previous work assumed that admissions are governed by the payment or nonpayment of a price, this paper allows for arbitrary admission rules. With sufficient inequality aversion, nondegenerate randomization in admissions is shown to be desirable for certain model specifications, with and without participation constraints. The paper also gives a sufficient condition on the distribution of preferences under which randomization is undesirable.
\end{abstract}

Keywords Utilitarian welfare maximization - Admission rules for excludable public goods $\cdot$ Randomization in optimal mechanisms

JEL Classification D61 $\cdot$ D63 $\cdot$ H21 $\cdot$ H41

\section{Introduction}

This paper studies the design of optimal utilitarian mechanisms for an excludable public good. Excludability provides a basis for making people pay to enjoy the public

It is a pleasure to acknowledge helpful comments from Felix Bierbrauer, Christoph Engel and Hendrik Hakenes, as well as five referees of this journal.

Electronic supplementary material The online version of this article (doi:10.1007/s00199-009-0488-3) contains supplementary material, which is available to authorized users.

M. F. Hellwig ( $\square)$

Max Planck Institute for Research on Collective Goods,

Kurt-Schumacher-Str. 10, 53113 Bonn, Germany

e-mail: hellwig@coll.mpg.de 
good. As discussed by Schmitz (1997) and Norman (2004), such payments can be used to finance the public good. As discussed by Hellwig (2005), such payments can also be used to provide for redistribution from people who get a lot of enjoyment out of the public good to people who get little enjoyment out of it.

If payment of a fee is required for admission, there must be exclusion of people for whom the benefits from the public good are not worth the admission fee. If there is nonrivalry in the enjoyment of the public good, such exclusion is inefficient. With incomplete information, however, the inefficiency may be outweighed by the benefits of obtaining funds for financing the public good or for redistribution.

In Hellwig (2005), I studied the use of exclusion to raise funds for redistribution under the assumption that exclusion is governed by an admission fee so that people who pay the fee are admitted and people who do not pay the fee are not admitted to the enjoyment of the public good. In contrast, Schmitz (1997) and Norman (2004) studied the use of exclusion to raise funds for public-goods finance without such an assumption and proved that, under a regularity condition on the distribution of preferences, an optimal mechanism is characterized by an admission fee. Other mechanisms, in particular, mechanisms that involve randomization in admissions, cannot be optimal.

Like Schmitz (1997) and Norman (2004), this paper studies the use of exclusion as a basis for raising funds without any prior assumption on the form of the incentive mechanism. In particular, it allows for mechanisms with nondegenerate randomization in admissions. Like Hellwig (2005), the paper allows for inequality aversion of the mechanism designer.

Mechanisms with nondegenerate randomization in admissions will in fact be shown to be optimal for certain model specifications. Under these mechanisms, there is a range of preference parameters where agents are given lotteries with admission probabilities that lie strictly between zero and one. Over the given range, admission probabilities as well as payments are strictly increasing functions of the preference parameters. These mechanisms with randomized admissions dominate the simple admission fee mechanisms in Hellwig (2005).

The finding that mechanisms with randomized admissions can dominate simple admission fee mechanisms is robust to the introduction of participation constraints. When participation constraints are imposed, admission fees are needed for publicgood finance as well as redistribution. Except for the inequality aversion of the mechanism designer, the mechanism design problem is the same as the problem studied by Schmitz (1997) and Norman (2004). For the same model specifications as before, in this problem, mechanisms with randomized admissions are again optimal. The finding of Schmitz (1997) and Norman (2004) that simple admission fee mechanisms are optimal is thus not robust to the introduction of (a sufficiently large degree of) inequality aversion of the mechanism designer.

In the examples given, the regularity condition that Schmitz (1997) and Norman (2004) had imposed on the cross-section distribution of the underlying preference parameter is always satisfied. The desirability of randomized admissions hinges on another feature of the cross-section distribution of preferences; in the examples where randomization is desirable the elasticity of the density of this distribution is decreasing in the preference parameter. The paper also shows that, if the elasticity of the density function is nondecreasing in the preference parameter, then a second-best mechanism 
is necessarily characterized by an admission fee, i.e., randomization in admissions is undesirable. Remarkably, the monotonicity condition on the density function is the same as the condition that Manelli and Vincent (2006) used to show that randomization is undesirable in the two-dimensional mechanism design problem of a profit-maximizing monopolist selling two goods to consumers with additively separable preferences when the preference parameters for the different goods are mutually independent.

In the following, Sect. 2 lays out the basic model and formulates the utilitarian welfare maximization problem. Section 3 shows that this problem has a unique solution and gives necessary and sufficient conditions for this solution. Section 4 discusses the equity-efficiency tradeoff and considers the dependence of optimal admission rules on the mechanism designer's inequality aversion. Section 5 shows that, for a parametrized set of model specifications, it is desirable to have randomized admissions. This section also shows that randomization is undesirable if the elasticity of the density function of the preference parameter distribution is nondecreasing in the preference parameter. Section 6 shows that, except for some obvious modifications, the results of the paper remain valid if interim participation constraints are imposed and admission fees are needed for public-good finance as well as redistribution. Section 7 discusses the robustness of the analysis to the introduction of risk aversion of the participants along with inequality aversion of the mechanism designer. Proofs are given either in the Appendix or in the Supplementary Material for the online version of this article; see also the Appendix to Hellwig (2009).

\section{The utilitarian mechanism design problem}

I study a large-economy version of the model considered in Schmitz (1997), Norman (2004), and Hellwig (2005). There are two goods in the economy, a private good and a public good, which is assumed to be excludable. The public good can be provided in one indivisible unit. ${ }^{1}$ People in the economy must determine a public-good provision level $Q \in\{0,1\}$ and, for each individual $h$ in the economy, a level $c^{h}$ of private-good consumption and a public-good admission decision $\chi^{h}$ where $\chi^{h}=1$ if the individual is admitted and $\chi^{h}=0$ if the individual is not admitted to the enjoyment of the public good. Given the triple $Q, c^{h}, \chi^{h}$, the individual obtains the payoff

$$
c^{h}+\theta^{h} \chi^{h} Q
$$

where $\theta^{h}$ is a parameter that determines the strength of his desire to enjoy the public good.

By an anonymity condition, the level $c^{h}$ of private-good consumption and the public-good admission decision $\chi^{h}$ depend on $h$ only through the taste parameter $\theta^{h}$ and through the realization $i^{h}$ of an exogenously given indicator variable $\tilde{l}^{h}$, which is introduced to allow for randomized allocations. An allocation is thus defined as a

\footnotetext{
1 This assumption is made for ease of exposition. Along the lines of Hellwig (2005), the analysis is easily extended to the case where the level of public-good provision is a continuous variable.
} 
triple $(Q, c(\cdot, \cdot), \chi(\cdot, \cdot))$ such that $Q$ is the level of public-good provision and, for each individual $h$ in the economy, one has

$$
c^{h}=c\left(\theta^{h}, i^{h}\right) \text { and } \chi^{h}=\chi\left(\theta^{h}, i^{h}\right) .
$$

For each $h$, the parameter $\theta^{h}$ is taken to be the consumer's private information. From the perspective of the other consumers, or of the system as a whole, $\theta^{h}$ is the realization of a random variable $\tilde{\theta}^{h}$, which takes values in the unit interval and has a probability distribution $F$ with a strictly positive, continuously differentiable density $f$. The random variable $\tilde{l}^{h}$ also takes values in the unit interval. It has a uniform distribution, denoted as $v$. The random variables $\tilde{\theta}^{h}$ and $\tilde{l}^{h}$ are independent, thus, knowing that $\tilde{\theta}^{h}=\theta^{h}$ does not provide the consumer with any information about $\tilde{l}^{h}$.

The distribution $F \times v$ of the random pair $\left(\tilde{\theta}^{h}, \tilde{l}^{h}\right)$ is assumed to be the same for all agents. I also assume that there is a large-numbers effect so that, with probability one, $F \times v$ is the cross-section distribution of the pair $\left(\theta^{h}, i^{h}\right)$ in the population. Indeed, I impose the stronger assumption that, with probability one, $F \times v$ is the cross-section distribution of the pair $\left(\theta^{h}, i^{h}\right)$ in any nonnegligible subset of the population.

These large-numbers effects justify the identification of anonymity with the restrictions that the level of public-good provision be a constant and that the outcome variables $c^{h}$ and $\chi^{h}$ for agent $h$ depend only on $\theta^{h}$ and $i^{h}$. If the cross-section distribution of preferences in the economy is the same in every state of nature, anonymity requires that the assessment of whether it is desirable to have the public good or not should be the same in every state of nature. If the cross-section distribution of preferences in any nonnegligible subset of the population is the same in every state of nature, then this distribution does not contain any information about the pair $\left(\tilde{\theta}^{h}, \tilde{l}^{h}\right)$ for any $h$, therefore, there is no scope for using such information in order to reduce the incentive effects of information about $\theta^{h}$ being private.

The reader who wonders about the underlying stochastic specification is referred to Sun (2006). For an atomless measure space of agents with random characteristics, Theorem 2.8, p. 39 in Sun (2006) shows that the cross-section distribution of characteristics in any subset of agents with positive measure satisfies the law of large numbers if and only if the following two conditions hold: (i) the random characteristics of different agents are essentially pairwise independent, i.e., for almost every agent $h$, the random pairs $\left(\tilde{\theta}^{h}, \tilde{l}^{h}\right)$ and $\left(\tilde{\theta}^{h^{\prime}}, \tilde{l}^{h^{\prime}}\right)$ are independent for almost every $h^{\prime} \neq h$; (ii) the function that maps agents and states into characteristics, here, the function $(h, \omega) \rightarrow\left(\tilde{\theta}^{h}(\omega), \tilde{l}^{h}(\omega)\right)$ is measurable with respect to a Fubini extension of the product measure that is obtained from the measure on the space of agents and the probability measure on the space of states. For any two measure spaces $(I, \mathcal{I}, \lambda)$ and $(\Omega, \mathcal{F}, P)$, the measure space $(I \times \Omega, \mathcal{W}, Q)$ is called a Fubini extension of the product $(I \times \Omega, \mathcal{I} \times \mathcal{F}, \lambda \times v)$ if and only if, for every $Q$-integrable real-valued function $f$ on $(I \times \Omega, \mathcal{W})$, the conclusions of Fubini's theorem hold with $(I, \mathcal{I}, \lambda)$ and $(\Omega, \mathcal{F}, P)$ as the relevant marginal spaces. ${ }^{2}$ The existence of a Fubini extension presumes that the underlying $\sigma$-algebra $\mathcal{I}$ on the space of agents is much richer than the

\footnotetext{
2 The existence of measure space specifications $(I, \mathcal{I}, \lambda)$ and $(\Omega, \mathcal{F}, P)$ that admit a Fubini extension is established in Sect. 5 of Sun (2006) (see also Sun and Zhang 2009 and Podczeck 2009).
} 
Borel or Lebesgue $\sigma$-algebra that has traditionally been used in specifying atomless measure spaces of agents. ${ }^{3}$

Allocations are assessed according to the functional

$$
\iint W(c(\theta, i)+\theta \chi(\theta, i) Q) \mathrm{d} \nu(i) \mathrm{d} F(\theta),
$$

where $W(\cdot)$ is a welfare function. The integral (2.3) represents the cross-section average (aggregate) of the welfare levels $W(c(\theta, i)+\theta \chi(\theta, i) Q)$ that are associated with the payoffs $c(\theta, i)+\theta \chi(\theta, i) Q$ of the different participants. The allocation problem is to maximize (2.3) over the set of admissible allocations. Admissibility will be defined in terms of feasibility and incentive compatibility. In some parts of the analysis, I will also impose a condition of individual rationality, i.e., a participation constraint.

The economy has an exogenously given per-capita production capacity that is equivalent to $Y$ units of private-good consumption. An allocation is feasible if the sum of aggregate private-good consumption and public-good provision costs does not exceed the available capacity, i.e., if

$$
\iint c(\theta, i) \mathrm{d} v(i) \mathrm{d} F(\theta)+K(Q) \leq Y
$$

where $K(Q)$ is the cost of providing the public good at the level $Q$. Because of nonrivalry in consumption, there are no costs to people enjoying the public good once it is installed.

An allocation is incentive-compatible if, for any $\theta \in[0,1]$, the expected payoff

$$
v(\theta):=\int c(\theta, i) \mathrm{d} v(i)+\theta Q \int \chi(\theta, i) \mathrm{d} v(i)
$$

of a consumer with preference parameter $\theta$ satisfies the inequality

$$
v(\theta) \geq \int c\left(\theta^{\prime}, i\right) \mathrm{d} v(i)+\theta Q \int \chi\left(\theta^{\prime}, i\right) \mathrm{d} v(i)
$$

for all $\theta^{\prime} \in[0,1]$. The allocation is individually rational if

$$
v(\theta) \geq Y
$$

\footnotetext{
${ }^{3}$ By having this richer structure of measurable sets, Sun avoids the well-known difficulties that arise in models with stochastic independence of agent characteristics when the space of agents is endowed with the Borel or Lebesgue $\sigma$-algebra. In such a model, with independence of the random variables $\tilde{\theta}^{h}$, for almost all states of nature $\omega$, the function $h \rightarrow \tilde{\theta}^{h}(\omega)$ that specifies each agent's preference parameter in the state $\omega$ is not measurable, and it is not clear what one means when one talks about the cross-section mean of the preference parameter. Treating such functions as elements of an uncountable-product space, Judd (1985) had suggested that a suitable extension of the induced measure on this space might yield a law of large numbers; he had also warned, however, that, by taking other extensions, one would obtain other versions of the model in which the law of large numbers did not apply. Feldman and Gilles (1985) showed that there is no one extension which provides a law of large numbers for all nonnegligible sets of agents (see also Khan and Sun 1999).
} 
for all $\theta$, i.e., if each agent is at least as well off as if the public good was not provided at all. This specification of individual rationality presumes that all agents have the same production capacity. By this presumption, I abstract from distributive concerns other than those that are due to heterogeneity in tastes for the public good.

The following assumptions are imposed throughout.

A.I The cost function $K(\cdot)$ satisfies $K(0)=0$ and $K(1)=\bar{K}$, where $0 \leq \bar{K}<$ $\max _{\theta} \theta(1-F(\theta))$.

A.II The welfare function $W(\cdot)$ in (2.3) is strictly increasing, strictly concave, and twice continuously differentiable.

Assumption A.I ensures that it is always desirable to have a positive level of public-good provision. Whereas an anonymous allocation without public-good provision provides people with the payoff $Y$, everybody can get a payoff greater than $Y$ if one sets $Q=1$ and, for some $p$,

$$
c(\theta)=Y-\bar{K}+p(1-F(p)) \text { and } \quad \chi(\theta, i)=0, \quad \text { if } \theta<p,
$$

and

$$
c(\theta)=Y-\bar{K}-p F(p) \text { and } \chi(\theta, i)=1, \quad \text { if } \theta \geq p .
$$

This allocation is obtained if admission to the public good is conditioned on the payment of a fee $p$, whereas people with $\theta<p$ do not ask for admission, people with $\theta \geq p$ ask for admission and pay the fee $p$. The allocation is obviously incentivecompatible and feasible. For any $\theta$, a person with taste parameter $\theta$ achieves the payoff

$$
v(\theta)=Y-\bar{K}+p(1-F(p))+\max (\theta-p, 0) .
$$

Under Assumption A.I, $p$ can be chosen so that $p(1-F(p))>\bar{K}$. For such $p$, one has $v(\theta)>Y$ for all $\theta$.

Assumption A.II expresses the notion that the planner is inequality-averse. If the preference parameters of the different consumers were publicly observable, he would thus choose an allocation satisfying $Q=1$ and $\chi(\theta, i)=1, c(\theta)=c(0)-\theta$ for all $\theta$ and $i$. Everybody would be admitted to the enjoyment of the public good, and the payoff levels $v(\theta)$ would all be equal to $v(0)=Y-\bar{K}+E \tilde{\theta}$.

However, this first-best allocation is not incentive compatible. If $\chi(\theta, i)=1$ and $c(\theta)=Y-\bar{K}+E \tilde{\theta}-\theta$ for all $\theta$ and $i$, then any consumer with $\theta>0$ has an incentive to understate his preference for the public good in order to raise his consumption of the private good without having to reduce his enjoyment of the public good.

The incompleteness of information calls for some compromise with first-best efficiency. Given the nonrivalry in consumption, efficiency considerations call for open admissions. With open admissions, however, payments cannot be conditioned on $\theta$. Then private-good consumption is the same for all agents, and agents with high $\theta$ are strictly better off than agents with low $\theta$. Indeed, agents with $\theta<\bar{K}$ are strictly worse off than they would be if the public good was not provided at all. Such an arrangement is incompatible with individual rationality. Even if individual rationality is not 
imposed, the unequal distribution of payoffs may give rise to equity concerns calling for some redistribution from agents with high $\theta$ to agents with low $\theta$. To provide for such redistribution, or to finance the public good at all if participation constraints are imposed, one needs to raise funds from agents with high $\theta$. For this purpose, there must be a threat of exclusion that discourages agents with high $\theta$ from claiming that they really do not care for the public good and therefore should not have to pay anything.

In the following, I first consider second-best mechanisms when participation constraints are not imposed. Subsequently, in Sect. 6, I will extend the analysis by imposing individual rationality, in addition to feasibility and incentive compatibility.

\section{Preliminary results}

For any $\theta$, let

$$
C(\theta)=\int c(\theta, i) \mathrm{d} \nu(i) \text { and } \pi(\theta)=\int \chi(\theta, i) \mathrm{d} \nu(i)
$$

be the expectations of $c\left(\tilde{\theta}^{h}, \tilde{l}^{h}\right)$ and $\chi\left(\tilde{\theta}^{h}, \tilde{l}^{h}\right)$ conditional on the information that $\tilde{\theta}^{h}=\theta$. The feasibility and incentive compatibility conditions (2.4)-(2.6) can then be rewritten as

$$
\begin{aligned}
& \int_{0}^{1} C(\theta) \mathrm{d} F(\theta)+K(Q) \leq Y, \\
& v(\theta)=C(\theta)+\theta \pi(\theta) Q,
\end{aligned}
$$

and

$$
v(\theta) \geq C\left(\theta^{\prime}\right)+\theta \pi\left(\theta^{\prime}\right) Q
$$

These conditions constrain the mechanism designer only with respect to $Q$ and the conditional-expectations functions $C(\cdot)$ and $\pi(\cdot)$, not with respect to the choice of $c(\cdot, \cdot)$ and $\chi(\cdot, \cdot)$ when $C(\cdot)$ and $\pi(\cdot)$ are taken as given.

The problem of choosing an admissible allocation to maximize (2.3) can therefore be decomposed into two steps. First, for any given $Q, C(\cdot)$, and $\pi(\cdot)$, the problem is to determine the optimal $c(\cdot, \cdot)$ and $\chi(\cdot, \cdot)$ subject to the constraint that (3.1) be satisfied for all $\theta$, for the stipulated $C(\theta)$ and $\pi(\theta)$. Second, the problem is to determine the optimal $Q, C(\cdot)$, and $\pi(\cdot)$. Because $W(\cdot)$ is strictly concave, the first of these steps is trivial. The mechanism designer chooses $c(\cdot, \cdot)$ and $\chi(\cdot, \cdot)$ so as to eliminate all remaining risk from people's payoffs. This observation yields:

Lemma 3.1 Let $Q, C(\cdot)$, and $\pi(\cdot)$ be given, and let $v(\cdot)$ be the associated expectedpayoff function. If $c(\cdot, \cdot)$ and $\chi(\cdot, \cdot)$ maximize (2.3) under the constraint that (3.1) be satisfied for all $\theta$, for the stipulated $C(\theta), \pi(\theta)$, then 


$$
c(\theta, i)=v(\theta)-\chi(\theta, i) \theta Q
$$

for $F \times v$-almost all $(\theta, i)$.

Given (3.5) and (3.3), the welfare functional (2.3) and the feasibility constraint (3.2) can be rewritten as

$$
\int_{0}^{1} W(v(\theta)) \mathrm{d} F(\theta)
$$

and

$$
\int_{0}^{1}[v(\theta)-Q \theta \pi(\theta)] \mathrm{d} F(\theta) \leq Y-K(Q) .
$$

Further, by standard arguments, due to Mirrlees (1976), the incentive compatibility conditions (3.3) and (3.4) are satisfied for all $\theta$ and $\theta^{\prime}$ if and only if $Q$ and the functions $v(\cdot), C(\cdot)$ and $\pi(\cdot)$ are such that $\pi(\cdot)$ is nondecreasing, and

$$
v(\theta)=v(0)+Q \int_{0}^{\theta} \pi(\eta) \mathrm{d} \eta
$$

for all $\theta \in[0,1] .^{4}$ The conditional expectation $\pi(\theta)$ of the admission indicator variable $\chi\left(\theta, \tilde{l}^{h}\right)$ is, of course, the probability that a person with preference parameter $\theta$ will be admitted to the enjoyment of the public good.

The problem of maximizing (2.3), subject to feasibility and incentive compatibility, is thus equivalent to the problem of choosing a public-good provision level $Q$, an expected-payoff function $v(\cdot)$, and a nondecreasing admission probability function $\pi(\cdot)$ so as to maximize (3.6) subject to (3.7) and the condition that $v(\cdot)$ satisfy (3.8) hold for all $\theta \in[0,1]$. I will refer to this latter problem as the reduced utilitarian problem.

As discussed above, Assumption A.I implies that an allocation without public-good provision is Pareto-dominated by an allocation with public-good provision financed by admission fees. This observation yields:

Lemma 3.2 Any solution to the reduced utilitarian problem satisfies $Q=1$.

For the record, I also state:

Proposition 3.3 The reduced utilitarian problem has a solution. The solution is unique up to modifications of $\pi(\cdot)$ at discontinuity points, which form a null set.

\footnotetext{
${ }^{4}$ See, e.g., Fudenberg and Tirole (1991, Chapter 7).
} 
Corollary 3.4 The problem of maximizing (2.3) over the set of feasible and incentivecompatible allocations has a solution. The solution is unique up to modifications

To get some insight into the nature of optimal solutions, I reformulate the reduced utilitarian problem so that the role of the admission probability function becomes clearer. If (3.8) is used to substitute for $v(\theta)$, with $Q=1$, the welfare functional (2.3) and the feasibility condition (3.2) take the form

$$
\int_{0}^{1} W\left(v(0)+\int_{0}^{\theta} \pi(\eta) \mathrm{d} \eta\right) \mathrm{d} F(\theta)
$$

and

$$
v(0) \leq Y-\bar{K}+\int_{0}^{1} \theta \pi(\theta) \mathrm{d} F(\theta)-\int_{0}^{1} \int_{0}^{\theta} \pi(\eta) \mathrm{d} \eta \mathrm{d} F(\theta) .
$$

According to (3.9), aggregate welfare depends on the base consumption $v(0)$ that is available to everybody and on the information rents $\int_{0}^{\theta} \pi(\eta) d \eta$ that people get according to their taste parameters. The admission probability rule $\pi(\cdot)$ determines the gross payoffs $\theta \pi(\theta)$ that people get from the enjoyment of the public good, the information rents $\int_{0}^{\theta} \pi(\eta) d \eta$, and, through (3.10), the maximum level of the base consumption $v(0)$ that is feasible.

Because of information rents, people with high $\theta$ have higher payoffs than people with low $\theta$. Because $W$ is strictly concave, therefore, some redistribution from the people with high $\theta$ to people with low $\theta$ would seem to be desirable. The question is which admission probability rule will best serve this purpose.

The effects of changes in $\pi(\theta)$ on the right-hand side of (3.10) are ambivalent: On the one hand, an increase in $\pi(\theta)$ raises the gross expected benefit $\theta \pi(\theta)$ of a person with taste parameter $\theta$ and therefore the amount that this person is willing to pay; on the other hand, this increase also raises the information rents $\int_{0}^{\bar{\theta}} \pi(\eta) d \eta$ of all persons with taste parameters $\bar{\theta}$ above $\theta$, lowering the amounts that they can be made to pay.

To study the resulting tradeoff, I use the control theoretic approach of Mirrlees (1971). Recalling that $F$ has a density $f$, I rewrite (3.6) and (3.7) as

$$
\int_{0}^{1} W(v(\theta)) f(\theta) \mathrm{d} \theta
$$

and

$$
\int_{0}^{1}[v(\theta)-\theta \pi(\theta)] f(\theta) \mathrm{d} \theta \leq Y-\bar{K}
$$


where I have also used $Q=1$. I also note that the incentive compatibility condition (3.8) is equivalent to the requirement that $v(\cdot)$ be absolutely continuous with (Radon-Nikodym) derivative $v^{\prime}(\cdot)$ satisfying

$$
v^{\prime}(\theta)=\pi(\theta)
$$

for almost all $\theta$. The reduced utilitarian problem is therefore equivalent to the problem of choosing $v(\cdot)$ and a nondecreasing function $\pi(\cdot)$ so as to maximize (3.11) subject to (3.12) and to (3.13) holding for almost all $\theta$. Except for the requirement that $\pi(\cdot)$ be nondecreasing (the second-order condition for incentive compatibility), this is a standard problem of optimal control with state variable $v$ and control variable $\pi$.

Again following Mirrlees (1971), I neglect the monotonicity condition on $\pi(\cdot)$ and study the relaxed utilitarian problem of maximizing (3.11) subject to only (3.12) and (3.13). If a solution to this problem happens to satisfy the monotonicity constraint on $\pi(\cdot)$, it is also a solution to the reduced utilitarian problem. ${ }^{5}$

The relaxed utilitarian problem is a standard optimal-control problem with Hamiltonian

$$
H=W(v(\theta)) f(\theta)+\lambda[(Y-\bar{K}-v(\theta)+\theta \pi(\theta)] f(\theta)+\varphi(\theta) \pi(\theta) .
$$

For this problem, standard arguments yield:

Proposition 3.5 The pair $(v(\cdot), \pi(\cdot))$ is a solution to the relaxed utilitarian problem if and only if there exist a scalar $\lambda$ and an absolutely continuous function $\varphi$ from $[0,1]$ into $\mathbb{R}$ such that

$$
\varphi(0)=\varphi(1)=0
$$

and, for almost all $\theta \in[0,1]$,

$$
\varphi^{\prime}(\theta)=-\left(W^{\prime}(v(\theta))-\lambda\right) f(\theta)
$$

and

$$
\pi(\theta) \in \arg \max _{\pi \in[0,1]} \pi[\lambda \theta f(\theta)+\varphi(\theta)]
$$

In this proposition, $\lambda$ is the Lagrange multiplier of the feasibility constraint (3.12); (3.16) provides the usual condition for the "dynamics" of the costate variable $\varphi$ that corresponds to the state variable $v$, and (3.15) the transversality conditions for $v$ at $\theta=0$ and $\theta=1$. By integration of (3.16), (3.15) and (3.16) are found to be equivalent to the requirements that

\footnotetext{
5 If a solution to the relaxed utilitarian problem fails to satisfy the monotonicity constraint on $\pi(\cdot)$, one must take recourse to the methods of Guesnerie and Laffont (1984) and Hellwig (2008). In the present context, there is little point in dealing with this complication.
} 


$$
\varphi(\theta)=\int_{\theta}^{1}\left[W^{\prime}(v(\eta))-\lambda\right] f(\eta) \mathrm{d} \eta
$$

for all $\theta \in[0,1]$ and

$$
\int_{0}^{1} W^{\prime}(v(\eta)) \mathrm{d} F(\eta)=\lambda
$$

Condition (3.17) reflects the maximum principle. Because the maximand is linear in $\pi$, this condition is equivalent to the Kuhn-Tucker conditions

$$
\begin{gathered}
\lambda \theta f(\theta)+\varphi(\theta) \leq 0 \quad \text { if } \pi(\theta)=0, \\
\lambda \theta f(\theta)+\varphi(\theta)=0 \quad \text { if } \pi(\theta) \in(0,1), \\
\lambda \theta f(\theta)+\varphi(\theta) \geq 0 \quad \text { if } \pi(\theta)=1 .
\end{gathered}
$$

\section{Inequality aversion and the equity-efficiency tradeoff}

Conditions (3.20)-(3.22) show that, for any $\theta$, the optimal choice of $\pi(\theta)$ depends on the sign of the expression

$$
g(\theta):=\lambda \theta f(\theta)+\varphi(\theta) .
$$

If $g(\theta)<0, \pi(\theta)$ must be zero; if $g(\theta)>0, \pi(\theta)$ must be one. If $\pi(\theta)$ is to take an intermediate value, the terms $\lambda \theta f(\theta)$ and $-\varphi(\theta)$ must just balance so that $g(\theta)=0$. The terms $\lambda \theta f(\theta)$ and $-\varphi(\theta)$ must also just balance, if $\theta$ is a point at which $\pi(\cdot)$ jumps from $\pi\left(\theta^{\prime}\right)=0$ for $\theta^{\prime}<\theta$ to $\pi\left(\theta^{\prime}\right)=1$ for $\theta^{\prime}>\theta{ }^{6}$

These conditions reflect an equity-efficiency tradeoff. This tradeoff has a similar structure as the equity-efficiency tradeoff in optimal utilitarian income taxation, e.g., equation (27) in Mirrlees (1971) or equation (6.17) in Hellwig (2007b). To gain some insight into its nature, I use (3.18) to write

$$
g(\theta)=\lambda \theta f(\theta)+\int_{\theta}^{1} W^{\prime}(v(\eta)) f(\eta) \mathrm{d} \eta-\lambda(1-F(\theta)) .
$$

The first term on the right-hand side of (4.2) corresponds to the allocative effect, the second and third terms to the distributive effect of a small increase in admission probabilities on a small interval above $\theta$.

If, for some small $\Delta>0$ and $\delta>0$, the admission probability is raised from $\pi\left(\theta^{\prime}\right)$ to $\pi\left(\theta^{\prime}\right)+\Delta$ for $\theta^{\prime} \in[\theta, \theta+\delta)$, there is an allocative effect because more people with

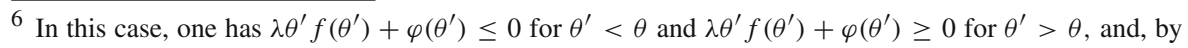
continuity, $\lambda \theta f(\theta)+\varphi(\theta)=0$.
} 
$\theta^{\prime} \in[\theta, \theta+\delta)$ obtain admission to the public good. This allocative effect provides an efficiency gain because the additional admissions involve no resource costs. Because private-good consumption satisfies (3.3), the gain takes mainly the form of additional revenues for the mechanism designer, an increase in the term $\int_{0}^{1} \theta \pi(\theta) d F(\theta)$ in (3.12). The amount of the increase is approximately $\Delta \int_{\theta}^{\theta+\delta} \theta^{\prime} d F\left(\theta^{\prime}\right) \approx \Delta \delta \theta f(\theta)$. The first term on the right-hand side of (4.2) corresponds to this effect, deflated by $\Delta \delta$.

If, for some small $\Delta>0$ and $\delta>0$, the admission probability is raised from $\pi\left(\theta^{\prime}\right)$ to $\pi\left(\theta^{\prime}\right)+\Delta$ for $\theta^{\prime} \in[\theta, \theta+\delta)$, there is also a distributive effect. As a result of the change, the information rent $\hat{v}\left(\theta^{\prime}\right)=\int_{0}^{\theta^{\prime}} \pi(\eta) d \eta$ of people with $\theta^{\prime}>\theta+\delta$ goes up. This raises the private-good consumption of people with $\theta^{\prime}>\theta+\delta$ and lowers the mechanism designer's revenues. The increase in private-good consumption of people with $\theta^{\prime}>\theta+\delta$ is equal to $\Delta \delta$ each. The decrease in the mechanism designer's is equal to $\Delta \delta(1-F(\theta))$. The effect on aggregate welfare is approximately equal to $\Delta \delta \int_{\theta}^{1} W^{\prime}(v(\eta)) f(\eta) d \eta-\lambda \Delta \delta(1-F(\theta))$ The second and third terms on the righthand side of (4.2) correspond to this effect, deflated by $\Delta \delta$.

Conditions (3.20)-(3.22) show that, if $\theta$ is a point of increase of $\pi(\cdot)$, the allocative and distributive effects of a small change in $\pi$ on a small interval $[\theta, \theta+\delta)$ just balance each other. If $\theta>0$, the allocative effect always involves a welfare gain. If $\theta \in(0,1)$, the distributive effect always involves a net welfare loss, i.e., the increase in information rents for people with $\theta^{\prime}>\theta+\delta$ is outweighed by the decrease in $v(0)$; whereas the reduction in $v(0)$ hits everybody, the increase in information rents only accrues to people with taste parameter above $\theta$ whose welfare weights on average are lower than the population average welfare weight. ${ }^{7}$

The size of the distributive effect and the tradeoff between the distributive effect and the allocative effect depend on the mechanism designer's inequality aversion. According to Atkinson (1973), a suitable measure of inequality aversion is provided by the relative curvature $\rho_{W}(v):=-\frac{W^{\prime \prime}(v)}{W^{\prime}(v)}$ of the welfare function. The following results characterize the solutions to the reduced utilitarian problem for extreme values of this measure. ${ }^{8}$

Proposition 4.1 There exists $A>0$ such that, if $\rho_{W}(v) \leq$ Afor all $v$, then the solution to the reduced utilitarian problem satisfies $\pi(\theta)=1$ and $v(\theta)=Y-\bar{K}+\theta$ for all $\theta$.

Proposition 4.2 Let $p_{M}=\min \arg \max _{\theta} \theta(1-F(\theta))$ be the smallest monopoly price, and let $\pi_{M}(\cdot)$ be the admission rule that people get admitted if and only if they pay $p_{M}$, i.e., $\pi_{M}(\theta)=0$ for $\theta \in\left[0, p_{M}\right)$ and $\pi_{M}(\theta)=1$ for $\theta \in\left(p_{M}, 1\right]$. If $\left\{W_{k}\right\}$ is a sequence of welfare functions such that $\lim _{k \rightarrow \infty} \rho_{W_{k}}(v)=\infty$, uniformly in $v$, the solutions $\left(v^{k}(\cdot), \pi^{k}(\cdot)\right)$ to the associated reduced utilitarian problems satisfy

\footnotetext{
7 Technically, the fact that the distributive effect involves a welfare loss is reflected in the negativity of the costate variable $\varphi(\theta)$ : By (3.15) and (3.16), the monotonicity of $v(\cdot)$, and the strict concavity of $W(\cdot)$, there exists $\bar{\theta} \in(0,1)$ so that, on the interval $(0, \bar{\theta}), \varphi^{\prime}(\theta)$ is negative and, on the interval $(\bar{\theta}, 1), \varphi^{\prime}(\theta)$ is positive, i.e., $\varphi$ is first decreasing from $\varphi(0)=0$ to $\varphi(\bar{\theta})<0$ and then increasing from $\varphi(\bar{\theta})<0$ to $\varphi(1)=1$.

${ }^{8}$ For mechanisms with nonrandom admission rules, these results had already been established in Hellwig (2005). Propositions 4.1 and 4.2 show that the conclusions remain valid if one allows for random as well as nonrandom admission rule.
} 


$$
\lim _{k \rightarrow \infty} v^{k}(\theta)=Y-\bar{K}+p_{M}\left(1-F\left(p_{M}\right)\right)+\max \left(\theta-p_{M}, 0\right)
$$

for all $\theta$, and

$$
\lim _{k \rightarrow \infty} \pi^{k}(\theta)=\pi_{M}(\theta)
$$

for all $\theta \neq p_{M}$.

Proposition 4.1 stands in contrast to findings on nonlinear income taxation which shows that, whenever there is inequality aversion, no matter how small it may be, it is always desirable to have at least some distortionary taxation as a basis for redistribution (see, e.g., Mirrlees 1971; Hellwig 2007b). Here, it is desirable to keep admissions completely open, i.e., to avoid any distortion if inequality aversion is uniformly small. The difference is due to the fact that, with nonrivalry in consumption, there is no cost to admitting an additional person to the enjoyment of the public good. If there was a variable cost $\int \gamma \pi(\theta) f(\theta) \mathrm{d} \theta$, with $\gamma>0$, of public-good enjoyment, this variable cost would have to be represented by an additional term $-\lambda \gamma \pi(\theta) f(\theta)$ in the Hamiltonian (3.14). The expression $g(\theta)$, which drives the choice of the admission probability $\pi(\theta)$, would then take the form $g(\theta)=\lambda(\theta-\gamma) f(\theta)+\varphi(\theta)$, which is strictly negative for all $\theta \leq \gamma$, and even for $\theta$ slightly above $\gamma$. The admission probability must then be zero for all $\theta \leq \gamma$, and even for $\theta$ slightly above $\gamma$. The allocative effect of a change in $\pi(\theta)$ is represented by the term $\lambda(\theta-\gamma) f(\theta)$, the distributive effect again by $\varphi(\theta)$. Both effects call for the exclusion of people with $\theta<\gamma$. For people with $\theta>\gamma$, the allocative effect calls for admission, the distributive effect for exclusion; with $\varphi(\gamma)<0$, the distributive effect prevails if $\theta>\gamma$ is sufficiently close to $\gamma$, and the allocative effect is small. With a population of mass $F(\theta)>0$ as net beneficiaries, for $\theta$ close to $\gamma$, the distributive effect is commensurate to the change in $\pi(\theta)$ and dominates the allocative effect. The optimal admission rule then involves some distortion even though the degree of inequality aversion may be very small. In contrast, in the absence of variable costs of people enjoying the public good, i.e., with $\gamma=0$, the distributive effect, as well as the allocative effect, of keeping out people with $\theta$ slightly above $\gamma$ is negligible. Which of the two effects dominates, depends on second-order considerations; these considerations in turn depend on the degree of inequality aversion.

At the other end of the spectrum, if inequality aversion is very large, it is desirable to provide the public good on terms similar to those of a profit-maximizing monopolist. Optimal utilitarian mechanisms converge to the optimal Rawlsian mechanism, which maximizes the payoff $v(0)$ of people with $\theta=0$, who are worst off. The Rawlsian mechanism manages the public good as a profit-maximizing monopolist would, charging the monopoly price $p_{M}$ in order to raise $v(0)$ to level $Y-\bar{K}+p_{M}\left(1-F\left(p_{M}\right)\right)$, the maximum that is at all feasible.

For an intermediate degree of inequality aversion, the optimal admission rule will lie between the open admissions of Proposition 4.1 and the Rawlsian admission rule. It is always desirable to be less restrictive than a profit-maximizing monopolist. 
Proposition 4.3 Regardless of $\rho_{W}(\cdot)$, the solution to the reduced utilitarian problem satisfies $\pi(\theta)=1$ for all $\theta>p_{M} \cdot{ }^{9}$

Propositions 4.1, 4.2, and 4.3 suggest that the optimal admission rule becomes more restrictive as the mechanism designer's inequality aversion goes up. For optimal mechanisms with nonrandom admission rules, this comparative-statics property is actually implied by Proposition 4.3 in Hellwig (2005). For the general case, allowing for randomized admissions, I do not have a proof, but can only formulate a conjecture. ${ }^{10}$

Conjecture 4.4 Let $W_{1}, W_{2}$ be two welfare functions such that $\rho_{W_{1}}(v)<\rho_{W_{2}}(v)$ for all $v$, and let $\left(v_{1}(\cdot), \pi_{1}(\cdot)\right),\left(v_{2}(\cdot), \pi_{2}(\cdot)\right)$ be the solutions to the associated reduced utilitarian problems. Then $v_{1}(0) \leq v_{2}(0)$ and $\pi_{1}(\theta) \geq \pi_{2}(\theta)$ for all $\theta$. Indeed, $v_{1}(0)<$ $v_{2}(0)$ and $\pi_{1}(\theta)>\pi_{2}(\theta)$ for some $\theta$ unless $v_{2}(0)=\bar{Y}-\bar{K}$ and $\pi_{2}(\theta)=1$ for all $\theta$.

\section{On the desirability of randomized admissions}

Turning to a more detailed analysis of the optimality conditions in Proposition 3.5, in this section, I discuss the desirability of having $\pi(\theta)$ lie strictly between zero and one for a nonnegligible set of $\theta$ s. The following proposition shows that it is not always desirable to simply charge a single admission fee and to admit people if and only if they pay the fee. For the given model specification, instead, the optimal admission rule involves randomized admissions.

Proposition 5.1 Assume that the welfare function $W$ and the density function $f$ are given as $W(v)=-\frac{1}{\rho} e^{-\rho v}$, where $\rho>0$, and $f(\theta)=A e^{-B \theta}$, where $B>0$ and $A=B /\left(1-e^{-B}\right)$. Then there exists a continuous function $\rho \rightarrow \hat{\theta}(\rho)$, taking values in the interval $\left[0, p_{M}\right)$, such that, for any $\rho>0$, the solution to the reduced utilitarian problem for the welfare function $W$ with inequality aversion $\rho$ satisfies

$$
\pi(\theta)=\frac{B}{\rho(2-B \theta)} \in(0,1) \text { if } \theta \in(0, \hat{\theta}(\rho)),
$$

and

$$
\pi(\theta)=1 \text { if } \theta \in(\hat{\theta}(\rho), 1] .
$$

\footnotetext{
${ }^{9}$ Strictly speaking, the proposition only shows that it is never desirable to be more restrictive than a profitmaximizing monopolist. However, using the first-order condition for the monopoly price $p_{M}$, one can show that, if the threshold for $\pi=1$ is shifted downwards from $p_{M}$ to a point $p$ just below $p_{M}$, the loss in admission fees revenues is small relative to the gain in information rents for people with $\theta>p_{M}$. I am not stating this formally because, in the absence of any information, apart from monotonicity, about the structure of $\pi(\cdot)$, the formal argument takes too much space. For the relaxed utilitarian problem, the claim is obvious from (4.2) showing that $g\left(p_{M}\right)>0$.

10 It is unsatisfactory to give a conjecture, rather than a theorem. In the theory of optimal nonlinear income taxation, however, we do not even have a conjecture as to what the appropriate analogue of the comparative-statics result of Roberts' (1977) for linear income taxation would be.
} 


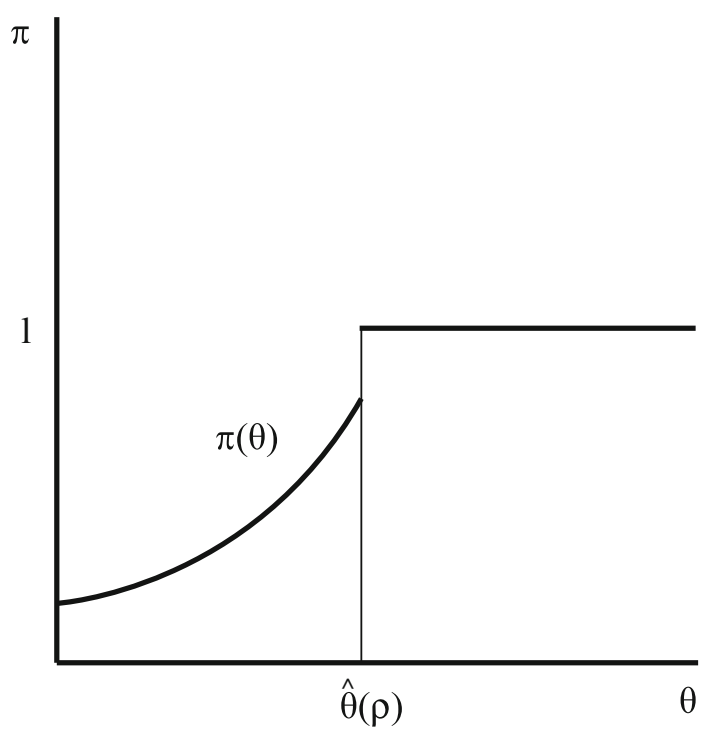

Fig. 1 Randomized admissions

Moreover, $\hat{\theta}(\rho)$ is strictly positive and increasing in $\rho$ if

$$
\frac{1}{2} \frac{1-e^{-B}}{B}>\frac{1-e^{-(\rho+B)}}{\rho+B}
$$

$\hat{\theta}(\rho)=0$ if (5.3) fails to hold.

For the case where the inequality aversion parameter $\rho$ is large enough to satisfy the inequality (5.3), the optimal admission rule (5.1), (5.2) is illustrated in Fig. 1. In this case, the optimal admission probability $\pi(\theta)$ is everywhere strictly positive. However, up to some critical value $\hat{\theta}(\rho)$, the optimal admission probability lies strictly between zero and one. Below $\hat{\theta}(\rho), \pi(\theta)$ is strictly increasing in $\theta$; at $\hat{\theta}(\rho)$, there is an upward jump from $\frac{B}{\rho(2-B \hat{\theta}(\rho))}$ to one.

Why should the admission rule $\pi(\cdot)$ take such a form? To answer this question, I note that the feasibility constraint (3.7) can be restated in terms of only the indirect utility function $v(\cdot)$. For this purpose, the term $\int_{0}^{1} \theta \pi(\theta) f(\theta) \mathrm{d} \theta$ in (3.7) is integrated by parts; with an appeal to the incentive compatibility condition (3.8), the terms $\int_{0}^{\theta} \pi(\eta) d \eta$ that appear are replaced by $v(\theta)-v(0)$. This yields

$$
\begin{aligned}
\int_{0}^{1} \theta \pi(\theta) f(\theta) \mathrm{d} \theta & =\left[\theta f(\theta) \int_{0}^{\theta} \pi(\eta) \mathrm{d} \eta\right]_{0}^{1}-\int_{0}^{1}\left(f(\theta)+\theta f^{\prime}(\theta)\right) \int_{0}^{\theta} \pi(\eta) \mathrm{d} \eta \mathrm{d} \theta \\
& =(v(1)-v(0)) f(1)-\int_{0}^{1}\left(f(\theta)+\theta f^{\prime}(\theta)\right) v(\theta) \mathrm{d} \theta+v(0) f(1) .
\end{aligned}
$$


Given (5.4), the feasibility constraint (3.7) can be rewritten as

$$
\int_{0}^{1} v(\theta)\left[2 f(\theta)+\theta f^{\prime}(\theta)\right] \mathrm{d} \theta-v(1) f(1) \leq Y-\bar{K} .
$$

The reduced utilitarian problem is thus equivalent to the problem of choosing $v(\cdot)$ to maximize (3.6) subject to (5.5) and subject to the requirement that $v(\cdot)$ be nondecreasing, convex and Lipschitz continuous with Lipschitz constant one. ${ }^{11}$

If one neglects the monotonicity, convexity, and Lipschitz continuity conditions on $v(\cdot)$ and just maximizes pointwise with respect to $v(\theta)$ for any $\theta$, one obtains the first-order condition

$$
W^{\prime}(v(\theta)) f(\theta)-\lambda\left[2 f(\theta)+\theta f^{\prime}(\theta)\right]=0,
$$

where $\lambda$ is again the Lagrange multiplier of the feasibility constraint. If the function $v(\cdot)$ that results from solving (5.6) for all $\theta$ happened to satisfy the requisite monotonicity, convexity, and Lipschitz continuity conditions, this function would actually be the solution to the reduced utilitarian problem. Of course, we know that, at least for $\theta \geq p_{M}$, the Lipschitz condition on $v(\cdot)$ must be strictly binding. However, if, locally, over some interval, the maximization condition (5.6) is compatible with the other constraints on $v(\cdot)$, then, over this interval, the solution to the reduced utilitarian problem must satisfy (5.6).

For the given welfare function $W$ and density function $f$, (5.6) can be rewritten as

$$
e^{-\rho v(\theta)}=\lambda(2-B \theta)
$$

which yields

$$
v(\theta)=-\frac{1}{\rho} \ln \lambda-\frac{1}{\rho} \ln (2-B \theta)
$$

and

$$
v^{\prime}(\theta)=\frac{B}{\rho(2-B \theta)} .
$$

(5.1) then follows from the incentive compatibility condition (3.8).

The rationale for randomized admissions here is different from the rationale for randomization that Stiglitz (1982) and Brito et al. (1995) give in the context of optimal income taxation. In that context, randomization is useful if it provides an incentive device to screen agents according to their earning abilities. This is only the case if agents with higher earning abilities exhibit greater risk aversion (Hellwig 2007a).

\footnotetext{
11 Monotonicity, convexity and the Lipschitz property ensure that the slope $v^{\prime}(\cdot)$ is nondecreasing and takes values in the unity interval.
} 
Here, the desirability of randomization has nothing to do with attitudes towards risk. It simply comes from the fact that the factors determining the choice of $v(\theta)$ for different $\theta$ may generate an indirect utility function with a slope $v^{\prime}$ that happens to lie strictly between zero and one. Its occurrence has more to do with the behavior of the density function $f$ than with the behavior of the welfare function $W$. Most importantly, the elasticity $\frac{\theta f^{\prime}(\theta)}{f(\theta)}=-B \theta$ of the density function in Proposition 5.1 is decreasing in $\theta$.

This monotonicity property of the elasticity $\frac{\theta f^{\prime}(\theta)}{f(\theta)}$ is actually necessary if the optimal admission rule is to involve some nondegenerate randomization. To see this, observe that, if $\pi(\theta) \in(0,1)$ over some interval, then one must have $g(\theta)=0$ on this interval. Hence also $g^{\prime}(\theta)=0$ on this interval, which is just (5.6). If $\pi(\theta)>0$, then, by the incentive compatibility condition $v^{\prime}(\theta)=\pi(\theta), v(\theta)$ is strictly increasing and, by the strict concavity of $W, W^{\prime}(v(\theta))$ is strictly decreasing in $\theta$. To balance the effect of $W^{\prime}(v(\theta))$ being strictly decreasing, the elasticity $\frac{\theta f^{\prime}(\theta)}{f(\theta)}$ must be decreasing in $\theta$ over this interval. Conversely, if the elasticity $\frac{\theta f^{\prime}(\theta)}{f(\theta)}$ is nondecreasing in $\theta$, then, quite generally, the optimal admission rule cannot involve any randomization. Thus, one obtains:

Proposition 5.2 If the elasticity $\frac{\theta f^{\prime}(\theta)}{f(\theta)}$ is nondecreasing in $\theta$, there exists $\hat{\theta} \in\left[0, p_{M}\right)$ such that the solution to the reduced utilitarian allocation problem satisfies

$$
\pi(\theta)=0 \text { if } \theta \in[0, \hat{\theta})
$$

and

$$
\pi(\theta)=1 \text { if } \theta \in[\hat{\theta}, 1]
$$

Moreover, $\hat{\theta}>0$ if and only if

$$
W^{\prime}(Y-\bar{K})>2 \int_{0}^{1} W^{\prime}(Y-\bar{K}+\theta) f(\theta) \mathrm{d} \theta
$$

As mentioned in Sect. 1, Manelli and Vincent (2006) use a similar monotonicity condition to ensure that a profit-maximizing monopolist does not want to use randomization in selling two goods to consumers with additively separable preferences. In their paper, the preference parameters $\theta_{1}, \theta_{2}$ for the different goods are assumed to be mutually independent, and the elasticity $\frac{\theta_{i} f_{i}^{\prime}\left(\theta_{i}\right)}{f_{i}\left(\theta_{i}\right)}$ of the density function of $\theta_{i}$ is assumed to be nondecreasing for each $i$.

I conclude this discussion with two remarks on the relation of Propositions 5.1 and 5.2 to the discussion in Sect.4. First, the two propositions give the same necessary and sufficient condition for not having completely open admissions: Condition (5.12) in Proposition 5.2 specializes to $(5.3)$ when $W^{\prime}(\cdot)$ and $f(\cdot)$ take the form specified in Proposition 5.1. To understand this condition, recall the optimality condition (3.17) for the choice of $\pi(\theta)$. For open admissions, i.e., $\pi(\theta)=1$ for all $\theta \in(0,1]$, to be 
optimal, one must have $g(\theta)=\lambda \theta f(\theta)+\varphi(\theta) \geq 0$ for all $\theta$, where $\lambda$ and $\varphi(\theta)$ are given by (3.19) and (3.18). By (3.19) and (3.18), one always has $g(0)=0$ and

$$
g^{\prime}(0)=\left(2 \lambda-W^{\prime}(v(0))\right) f(0)
$$

If $g(\theta)$ is to be nonnegative for $\theta$ close to zero, one must therefore have

$$
2 \lambda \geq W^{\prime}(v(0))
$$

Under open admissions, $v(\theta)=Y-\bar{K}+\theta$ for all $\theta$, so, by (3.19), (5.14) takes the form

$$
\int_{0}^{1} W^{\prime}(Y-\bar{K}+\theta) f(\theta) \mathrm{d} \theta \geq W^{\prime}(Y-\bar{K}),
$$

which is the negation of (5.12). Conversely, (5.12) implies that there is a preference to restrict admissions for $\theta$ close to zero. Thus, (5.15) is necessary for open admissions to be optimal. Under the additional assumptions of Propositions 5.1 and 5.2, this condition is also sufficient for the optimality of open admissions.

Second, Conjecture 4.4 is true in the setting of Proposition5.1 as well as Proposition 5.2. In both settings, optimal admission rules become more restrictive if inequality aversion goes up. For Proposition 5.2, where optimal admission rules do not involve randomization, this claim follows from Proposition 4.3 in Hellwig (2005). For Proposition 5.1, the claim follows from the fact that the threshold $\hat{\theta}(\rho)$ is increasing in $\rho$, i.e., the interval with $\pi(\theta)<1$ becomes larger as $\rho$ goes up, and, moreover, by (5.1), for any $\theta<\hat{\theta}(\rho)$, the admission probability $\pi(\theta)$ is decreasing in $\rho$.

\section{The model with participation constraints}

This last part of the paper shows that, apart from some obvious changes, the preceding analysis is robust to the imposition of participation constraints. This is true, in particular, of the findings that randomization may be desirable if the elasticity $\frac{\theta f^{\prime}(\theta)}{f(\theta)}$ is decreasing, and is never desirable if the elasticity $\frac{\theta f^{\prime}(\theta)}{f(\theta)}$ is increasing in $\theta$.

Because, by incentive compatibility, the indirect utility function is nondecreasing in $\theta$, the participation constraint (2.7) can be reduced to the inequality

$$
v(0) \geq Y
$$

The imposition of this constraint, in addition to feasibility and incentive compatibility, obviously has no effect on the validity of Lemmas 3.1 and 3.2 and of Proposition 3.3.

The reduced utilitarian problem with participation constraints then is to choose an indirect utility function $v(\cdot)$ and a nondecreasing function $\pi(\cdot)$ so as to maximize (3.6) subject to (6.1), (3.12), and to (3.13) holding for almost all $\theta$. The relaxed utilitarian problem with participation constraints is the problem of maximizing (3.6) subject 
only to (6.1), (3.12), (3.13), without the monotonicity condition on $\pi(\cdot)$. Except for the transversality condition for $v(0)$, the characterization given in Proposition 3.5 remains valid for the relaxed utilitarian problem with participation constraints. The transversality condition $\varphi(0)=0$ in Proposition 3.5 is replaced by the new transversality condition

$$
\varphi(0) \leq 0 \text { and } \varphi(0)(v(0)-Y)=0 .
$$

Because of this change, the Lagrange multiplier for the feasibility constraint is no longer given by (3.19). With $\varphi$ again satisfying the differential equation (3.16) and the boundary condition $\varphi(1)=0,(6.2)$ is equivalent to the requirement that

$$
\lambda \geq \int_{0}^{1} W^{\prime}(v(\theta)) f(\theta) \mathrm{d} \theta,
$$

with equality if $v(0)>Y$.

If one combines the participation constraint (6.1) with the feasibility constraint (3.10), one obtains the inequality

$$
\bar{K} \leq \int_{0}^{1} \theta \pi(\theta) \mathrm{d} F(\theta)-\int_{0}^{1} \int_{0}^{\theta} \pi(\eta) \mathrm{d} \eta \mathrm{d} F(\theta),
$$

requiring that the cost of public-good provision be covered by the share of the benefits from the public good that is appropriated by the mechanism designer. The participation constraint $v(0) \geq Y$ eliminates the possibility of financing the public good by a lump sum tax. As discussed by Schmitz (1997) and Norman (2004), the requisite revenue must come from admission fees.

Condition (6.4) is obviously incompatible with the open-admissions rule of Proposition 4.1. For low levels of inequality aversion, the open-admissions rule is therefore replaced by a rule stipulating an admission fee at which revenues just cover the cost of public-good provision. Assumption A.I guarantees that such a fee exists and that it is smaller than $p_{M}$. In deference to Dupuit (1844), I will refer to this fee as the Dupuit fee and the associated admission rule as the Dupuit admission rule. Formally, the Dupuit fee is given as the smallest solution to the equation ${ }^{12}$

$$
\bar{\theta}(1-F(\bar{\theta}))=\bar{K}
$$

The following result provides the analogue of Proposition 4.1 for the utilitarian problem with participation constraints.

\footnotetext{
${ }^{12}$ For an admission rule characterized by an admission fee $\bar{\theta}$, the right-hand side of (6.4) takes the form $\int_{\bar{\theta}}^{1} \theta \mathrm{d} F(\theta)-\int_{0}^{1} \max (\theta-\bar{\theta}, 0) \mathrm{d} F(\theta)=\bar{\theta}(1-F(\bar{\theta})$.
} 
Proposition 6.1 Assume that the map $\theta \rightarrow \frac{\theta f(\theta)}{1-F(\theta)}$ is increasing, with a derivative that is bounded away from zero on $(0,1)$. Then, there exists $A>0$ such that, if $\rho_{W}(v) \leq A$ for all $v$, the solution to the reduced utilitarian problem with participation constraints $i$ given by the Dupuit admission rule, with $v$ satisfying $v(\theta)=Y+\max (0, \theta-\bar{\theta})$ for all $\theta$.

The requirement that the ratio $\frac{\theta f(\theta)}{1-F(\theta)}$ be increasing in $\theta$ is the condition that Schmitz (1997) and Norman (2004) impose to obtain the optimality of the Dupuit admission rule in the absence of inequality aversion. By comparison to their papers, the assumption that the derivative of this ratio be bounded away from zero provides for a slight strengthening of this requirement. The purpose of this strengthening is to avoid the possibility that the derivative of $\frac{\theta f(\theta)}{1-F(\theta)}$ with respect to $\theta$ is equal to zero precisely at the Dupuit fee $\bar{\theta}$, in which case the tradeoff between the allocative and distributive effects of an increase in the admission fee above $\bar{\theta}$ is difficult to disentangle even though inequality aversion is small.

At the other end of the spectrum, when inequality aversion is large, Proposition 4.2 implies that the inequality (6.1) is automatically satisfied, even when it is not imposed as a constraint. In the case, the participation constraint is not binding, and the results of the preceding analysis carry over without change. In particular, one obtains the following analogue of Proposition 5.1:

Proposition 6.2 Assume that the welfare function $W$ and the density function $f$ are given as $W(v)=-\frac{1}{\rho} e^{-\rho v}$, where $\rho>0$, and $f(\theta)=A e^{-B \theta}$, where $B>0$ and $A=B /\left(1-e^{-B}\right)$. Then there exist continuous functions $\rho \rightarrow \bar{\theta}(\rho)$ and $\rho \rightarrow \theta^{*}(\rho) \leq$ $\bar{\theta}(\rho)$ that take values in the interval $\left[0, p_{M}\right)$, such that, for any $\rho>0$, the solution to the reduced utilitarian problem with participation constraints for the welfare function $W$ with inequality aversion $\rho$ satisfies

$$
\begin{aligned}
& \pi(\theta)=0 \quad \text { if } \theta \in\left[0, \theta^{*}(\rho)\right) \\
& \pi(\theta)=\frac{B}{\rho(2-B \theta)} \in(0,1) \text { if } \theta \in\left(\theta^{*}(\rho), \bar{\theta}(\rho)\right),
\end{aligned}
$$

and

$$
\pi(\theta)=1 \text { if } \theta \in(\bar{\theta}(\rho), 1] .
$$

Moreover, for any $\rho, \bar{\theta}(\rho)$ is the larger of the Dupuit admission fee $\bar{\theta}$ and the critical $\hat{\theta}(\rho)$ in Proposition 5.1. $\theta^{*}(\rho)$ is nonincreasing in $\rho$, with $\theta^{*}(\rho)=\bar{\theta}(\rho)$ if $\bar{\theta}(\rho)=\bar{\theta}$ and $\theta^{*}(\rho)=0$ if the solution to the reduced utilitarian problem in Proposition 5.1 satisfies (6.1) automatically. In the intermediate case, if $\bar{\theta}(\rho)>\bar{\theta}$ and the participation constraint (6.1) is strictly binding, $\theta^{*}(\rho)$ lies strictly between zero and $\bar{\theta}(\rho)$.

Figure 2 illustrates the optimal admission rule of Proposition 6.2 for the intermediate case where $\rho$ is both, high enough so that the critical $\hat{\theta}(\rho)$ in Proposition 5.1 exceeds the Dupuit admission fee $\bar{\theta}$, and low enough so that the participation constraint (6.1) is strictly binding. In this case, the admission rule is characterized 


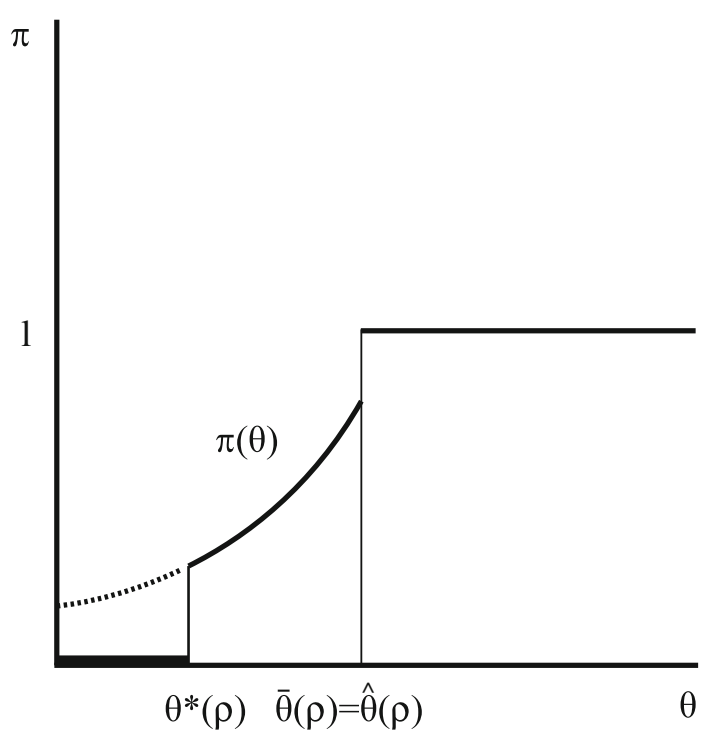

Fig. 2 Randomized admissions with participation constraints

by two thresholds $\theta^{*}(\rho)$ and $\bar{\theta}(\rho)$. Below $\theta^{*}(\rho)$, the lower threshold, admission probabilities are zero. Above $\theta^{*}(\rho)$, admission probabilities are the same as in the absence of participation constraints, taking the values $\frac{B}{\rho(2-B \theta)}$ between $\theta^{*}(\rho)$ and $\bar{\theta}(\rho)$ and the value one above $\bar{\theta}(\rho)$; the upper threshold is the same as the threshold $\hat{\theta}(\rho)$ in Proposition 5.1. Thus, in particular, this threshold is independent of $\bar{K}$.

The lower threshold $\theta^{*}(\rho)$ is determined so that admission fee revenues cover the cost of public-good provision. By fully excluding people with $\theta<\theta^{*}(\rho)$, the mechanism designer obtains a greater share of the benefits enjoyed by people with $\theta>\theta^{*}(\rho)$. This is necessary if $\rho$ is low enough so that the participation constraint (6.1) is strictly binding. However, if $\hat{\theta}(\rho)>\bar{\theta}$, there is no need to push $\theta^{*}(\rho)$ all the way up to $\bar{\theta}(\rho))=\hat{\theta}(\rho)$. In this case, there is still a nondegenerate interval $\left(\theta^{*}(\rho), \bar{\theta}(\rho)\right)$ in which optimal admission probabilities lie strictly between zero and one.

To conclude this section, I note that, by the same arguments as before, with participation constraints as well as without, randomization in admissions is not optimal if the elasticity $\frac{\theta f^{\prime}(\theta)}{f(\theta)}$ is nondecreasing in $\theta$. Formally, one obtains:

Proposition 6.3 If the elasticity $\frac{\theta f^{\prime}(\theta)}{f(\theta)}$ is nondecreasing in $\theta$, there exists $\hat{\theta}^{*} \in\left[0, p_{M}\right)$ such that the solution to the reduced utilitarian allocation problem with participation constraints satisfies

$$
\pi(\theta)=0 \text { if } \theta \in\left[0, \hat{\theta}^{*}\right),
$$

and

$$
\pi(\theta)=1 \text { if } \theta \in\left[\hat{\theta}^{*}, 1\right] .
$$


Moreover, $\hat{\theta}^{*}$ is the larger of the Dupuit admission fee $\bar{\theta}$ and the critical $\hat{\theta}$ in Proposition 5.2.

\section{Risk aversion}

Two referees have suggested that, in the present context, the assumption of risk neutrality consumers is problematic — not just because risk neutrality is special, but because the analysis is concerned with risk randomization and risk. The treatment of incomplete information rests on a model involving prior uncertainty about each individual's preference parameter $\theta$. From an ex ante point of view, the assessment of a given allocation by a consumer is likely to depend on his risk attitudes. Therefore, it is important that the conclusions of the analysis should be robust to the introduction of risk aversion.

Risk aversion of consumers affects the utilitarian allocation problem in two ways. First, it introduces an additional element of inequality aversion. Even if the welfare function $W(\cdot)$ was linear, equity considerations might play a role as way of reducing the consequences of ex ante uncertainty about preferences. Second, risk aversion of consumers affects the incidence of incentive constraints. If people with high $\theta$ are less willing to accept a given risk in private-good consumption than people with low $\theta$, it may be desirable to have some randomization over the private-good consumption of people with low $\theta$ in order to alleviate incentive constraints for people with high $\theta$. This corresponds to the suggestion of Stiglitz (1982) and Brito et al. (1995) that randomization in income taxation may be useful as way of alleviating incentive constraints for high-productivity people.

Since this is hardly the point to begin a new paper, I only make a few remarks on the implications of allowing for risk aversion of consumers. If risk aversion is uncorrelated with the hidden characteristic, there is no point in using such randomization as an incentive device. For example, if the von Neumann-Morgenstern utility function takes the form

$$
u(c(\theta, i)+\chi(\theta, i) \theta Q) \equiv-\frac{1}{\delta} e^{-\delta(c(\theta, i)+\chi(\theta, i) \theta Q)}
$$

for some $\delta>0$, attitudes towards risks in private-good consumption are independent of $\theta$. In this case, randomization in private-good consumption, conditional on $\theta$ and on the public-good admission decision, can only reduce expected payoffs without alleviating incentive constraints. ${ }^{13}$ Attention can therefore be restricted to allocations with private-good consumption taking the form

$$
c(\theta, i)=c_{0}(\theta) \text { if } \chi(\theta, i)=0 \text { and } c(\theta, i)=c_{1}(\theta) \text { if } \chi(\theta, i)=1 .
$$

\footnotetext{
13 As in Hellwig (2007a), this conclusion can also be obtained if participants exhibit nonincreasing, rather than constant absolute risk aversion.
} 
In the absence of participation constraints, the utilitarian mechanism design problem then is to choose functions $c_{0}(\cdot), c_{1}(\cdot), \pi(\cdot)$, as well as a public-good provision level $Q$, so as to maximize the welfare functional ${ }^{14}$

$$
\int_{0}^{1}\left[\pi(\theta) W\left(-\frac{1}{\delta} e^{-\delta\left(c_{1}(\theta)+\theta Q\right)}\right)+(1-\pi(\theta)) W\left(-\frac{1}{\delta} e^{-\delta c_{0}(\theta)}\right)\right] f(\theta) \mathrm{d} \theta
$$

under the feasibility constraint

$$
\int_{0}^{1}\left[\pi(\theta) c_{1}(\theta)+(1-\pi(\theta)) c_{0}(\theta)\right] f(\theta) \mathrm{d} \theta \leq Y-K(Q)
$$

and the incentive compatibility condition that

$$
v(\theta) \geq-\frac{1}{\delta} \pi\left(\theta^{\prime}\right) e^{-\delta\left(c_{1}\left(\theta^{\prime}\right)+\theta Q\right)}-\frac{1}{\delta}\left(1-\pi\left(\theta^{\prime}\right)\right) e^{-\delta c_{0}\left(\theta^{\prime}\right)}
$$

for all $\theta$ and $\theta^{\prime}$ where

$$
v(\theta)=-\frac{1}{\delta} \pi(\theta) e^{-\delta\left(c_{1}(\theta)+\theta Q\right)}-\frac{1}{\delta}(1-\pi(\theta)) e^{-\delta c_{0}(\theta)} .
$$

As before, for any $\theta, \pi(\theta):=\int \chi(\theta, i) \mathrm{d} \nu(i)$.

Using the arguments of Mirrlees (1976), one can show that the first-order and second-order necessary conditions for incentive compatibility are jointly sufficient, as well as necessary, for incentive compatibility. The first-order condition requires that

$$
v^{\prime}(\theta)=Q \pi(\theta) e^{-\delta\left(c_{1}(\theta)+\theta Q\right)}
$$

for all $\theta$, the second-order condition requires that the function $\theta \rightarrow Q \pi(\theta) e^{-\delta c_{1}(\theta)}$ be nondecreasing. The utilitarian mechanism design problem then is to choose $Q, c_{0}(\cdot), c_{1}(\cdot), \pi(\cdot)$ and the expected-payoff function $v(\cdot)$ so as to maximize (7.3) subject to (7.4), (7.5), (7.6), and the requirement that $Q \pi(\theta) e^{-\delta c_{1}(\theta)}$ be nondecreasing in $\theta$. The corresponding relaxed utilitarian problem is to maximize (7.3) subject to (7.4), (7.5), (7.6), without the monotonicity requirement on $Q \pi(\theta) e^{-\delta c_{1}(\theta)}$.

This relaxed utilitarian problem can again be analysed by control theoretic methods. This yields the following:

- In contrast to Lemma 3.1, it is undesirable to set $c_{1}(\theta)=c_{0}(\theta)-\theta$. The reason is the appearance of $c_{1}(\theta)$ in the incentive constraint (7.6), for which there is analogue in the case of risk neutrality. If the curvature $\rho_{W}(v(\theta))$ of the welfare function is

\footnotetext{
14 This welfare specification is based on a strictly consequentialist approach. An alternative approach would specify welfare as a function of participants' payoff expectations rather than payoff realizations. The analysis would hardly be changed.
} 
everywhere equal to zero, i.e., if there is no inequality aversion, it is actually desirable to set $c_{1}(\theta)=c_{0}(\theta)$. If there is inequality aversion as well as risk aversion, it is desirable to have $c_{0}(\theta)-\theta<c_{1}(\theta)<c_{0}(\theta)$.

- The results of Sect. 4 go through unchanged, except that, in these results, the curvature of $W$ must be replaced by the curvature

$$
\rho_{V}(c)=\rho_{W}(u(c))+\rho_{u}(c)
$$

of the composition $V=W \circ u$. The consumers' risk aversion $\rho_{u}(c)=\delta$ is simply added to the inequality aversion inherent in the welfare function $W$.

- If the risk aversion coefficient $\delta$ is close to zero, optimal admission rules must be close to the (unique) optimal admission rule in the case of risk neutrality. For the welfare and density functions specified in Proposition 5.1, there must therefore be randomization in admissions if the parameters $\rho$ and $B$ satisfy the inequality (5.3) and $\delta$ is sufficiently small. Whether randomization can be desirable for arbitrary $\delta$, regardless of what the curvature of $W$ might be is at this point is an open question.

\section{Appendix A: Proofs}

Lemmas 3.1 and 3.2 follow directly from the arguments given in the text.

Proposition 3.3 is proved in the supplementary material to the online version of this paper, see also Appendix A.1 to Hellwig (2009).

As for Proposition 3.5, the "only if" part of this proposition follows from Clarke (1983) version of the Maximum Principle, the "if" part from the argument of Mangasarian (1966). The details are left to the reader.

Propositions 4.1-4.3 are proved in the supplementary material to the online version of this paper, see also Appendix A.2 in Hellwig (2009) and the proofs of the analogous results (Propositions 4.1, 4.2, and 3.3) in Hellwig (2005).

Turning to the proof of Proposition 5.1, suppose that the welfare function $W$ and the density function $f$ take the form assumed in this proposition, i.e., $W(v \mid \rho)=-\frac{1}{\rho} e^{-\rho v}$ and $f(\theta)=A e^{-B \theta}$, where $A=\frac{B}{1-e^{-B}}$. The following properties of the distribution function $F$ and the density function $f$ will be repeatedly used.

- The revenue function

$$
\theta \rightarrow \theta(1-F(\theta))=\theta \frac{e^{-B \theta}-e^{-B}}{1-e^{-B}}
$$

has first derivative

$$
\frac{e^{-B \theta}(1-B \theta)-e^{-B}}{1-e^{-B}}
$$

and second derivative

$$
\frac{-B e^{-B \theta}(2-B \theta)}{1-e^{-B}}
$$


- By (A.2) and (A.3), the revenue function is strictly quasi-concave.

- There a unique revenue-maximizing price $p_{M}$. It satisfies

$$
e^{-B p_{M}}\left(1-B p_{M}\right)-e^{-B}=0 .
$$

- For all $\theta \in\left[0, p_{M}\right)$,

$$
e^{-B \theta}(1-B \theta)-e^{-B}>0
$$

- For all $\theta \in\left[0, p_{M}\right]$,

$$
2-B \theta>0 \text {. }
$$

A central role in the analysis will be played by the function $H(\cdot)$ that is defined by

$$
H(\hat{\theta}):=\int_{0}^{\hat{\theta}}(2-B \theta) A e^{-B \theta} \mathrm{d} \theta+(2-B \hat{\theta}) e^{\rho \hat{\theta}} \int_{\hat{\theta}}^{1} A e^{-(\rho+B) \theta} \mathrm{d} \theta
$$

By integration, one can also write

$$
H(\hat{\theta})=\frac{A}{B}\left(1-e^{-B \hat{\theta}}(1-B \hat{\theta})\right)+(2-B \hat{\theta}) A e^{-B \hat{\theta}} \frac{1-e^{-(\rho+B)(1-\hat{\theta})}}{\rho+B},
$$

or, because $A=\frac{B}{1-e^{-B}}$,

$$
H(\hat{\theta})=1-\frac{A}{B}\left(e^{-B \hat{\theta}}(1-B \hat{\theta})-e^{-B}\right)+(2-B \hat{\theta}) A e^{-B \hat{\theta}} \frac{1-e^{-(\rho+B)(1-\hat{\theta})}}{\rho+B} .
$$

Finally, the first and second derivatives of $H(\cdot)$ are given as

$$
H^{\prime}(\hat{\theta})=[\rho(2-B \hat{\theta})-B] e^{\rho \hat{\theta}} \int_{\hat{\theta}}^{1} A e^{-(\rho+B) \theta} \mathrm{d} \theta
$$

and

$$
H^{\prime \prime}(\hat{\theta})=-\rho B e^{\rho \hat{\theta}} \int_{\hat{\theta}}^{1} A e^{-(\rho+B) \theta} \mathrm{d} \theta+\left[\rho-\frac{A e^{-(\rho+B) \hat{\theta}}}{\int_{\hat{\theta}}^{1} A e^{-(\rho+B) \theta} \mathrm{d} \theta}\right] H^{\prime}(\hat{\theta}) .
$$


With this preparation, I now turn to the proof of Proposition 5.1. The following lemma specifies the critical $\hat{\theta}(\rho)$.

Lemma A.1 For any $\rho \in \mathbb{R}_{++}$, there exists $\hat{\theta}(\rho) \in\left[0, p_{M}\right)$ such that

$$
\hat{\theta} \in\left(\hat{\theta}(\rho), p_{M}\right] \text { implies } H(\hat{\theta})>1
$$

and

$$
\hat{\theta} \in[0, \hat{\theta}(\rho)) \text { implies } H(\hat{\theta})<1 \text {; }
$$

$\hat{\theta}(\rho)>0$ if

$$
1>2 A \frac{1-e^{-(\rho+B)}}{\rho+B},
$$

and $\hat{\theta}(\rho)=0$ if

$$
1 \leq 2 A \frac{1-e^{-(\rho+B)}}{\rho+B},
$$

The map $\rho \rightarrow \hat{\theta}(\rho)$ is nondecreasing on $\mathbb{R}_{+}$; it is strictly increasing on the set of $\rho$ satisfying (A.13); $\lim _{\rho \rightarrow \infty} \hat{\theta}(\rho)=p_{M}$.

Proof By (A.11), $H^{\prime}(\bar{\theta})=0$ implies that

$$
H^{\prime}(\hat{\theta}) \gtreqless 0 \quad \text { as } \hat{\theta} \lesseqgtr \bar{\theta}
$$

Thus, $H(\cdot)$ is strictly quasi-concave.

From (A.4), (A.6), and (A.9), $H\left(p_{M}\right)>1$. If $H(0) \geq 1$, then, because $H(\cdot)$ is strictly quasi-concave, it follows that $H(\hat{\theta})>1$ for all $\hat{\theta} \in\left(0, p_{M}\right]$. In this case, (A.12) and (A.13) are true for $\hat{\theta}(\rho)=0$. If $H(0)<1$, then, by the intermediate-value theorem, there exists $\theta^{*} \in\left(0, p_{M}\right)$ such that $H\left(\theta^{*}\right)=1$. Moreover, because $H(\cdot)$ is strictly quasi-concave, one must have $H(\hat{\theta})>1$ for $\hat{\theta} \in\left(\theta^{*}, p_{M}\right]$ and $H(\hat{\theta})<1$ for $\hat{\theta} \in\left[0, \theta^{*}\right)$. In this case, (A.12) and (A.13) are true for $\hat{\theta}(\rho)=\theta^{*}$.

From (A.9), one has

$$
H(0)-1=-1+2 A \frac{1-e^{-(\rho+B)}}{\rho+B} .
$$

Thus, (A.14) implies $H(0)<1$, and (A.15) implies $H(0) \geq 1$.

Because $H(\cdot)$ is increasing at $\hat{\theta}(\rho)$, strict monotonicity of $\hat{\theta}(\cdot)$ on the set of $\rho$ satisfying (A.14) follows from the observation that, the right-hand side of (A.9) is decreasing in $\rho$. Global weak monotonicity follows from the observation that the ratio $\frac{1-e^{-(\rho+B)}}{\rho+B}$ is decreasing in $\rho$ so that (A.15) holds if $\rho$ is small and (A.14) holds if $\rho$ is large. 
The next lemma gives a condition under which it is optimal to set $\pi(\theta)=1$ above some threshold $\hat{\theta} \geq \hat{\theta}(\rho)$. The analysis turns on the optimality conditions of Proposition 3.5. For the given model specification, the expression $g(\theta)$ in (4.2), which drives the choice of $\pi(\theta)$, takes the form

$$
g(\theta)=\int_{\theta}^{1} e^{-\rho v(\eta)} A e^{-B \eta} \mathrm{d} \eta-\lambda \frac{A}{B}\left(e^{-B \theta}(1-B \theta)-e^{-B}\right) .
$$

If $\pi(\theta)=1$ above some threshold $\hat{\theta}$, then, because $v^{\prime}(\eta)=\pi(\eta)$ for all $\eta$, one has $v(\eta)=v(\hat{\theta})+\eta-\hat{\theta}$ for all $\eta>\hat{\theta}$, and (A.17) takes the form

$$
\begin{aligned}
g(\theta)= & e^{-\rho v(\hat{\theta})} A e^{-B \hat{\theta}} \frac{1-e^{-(\rho+B)(1-\hat{\theta})}}{\rho+B} \\
& -\lambda \frac{A}{B}\left(e^{-B \theta}(1-B \theta)-e^{-B}\right) .
\end{aligned}
$$

Lemma A.2 Let $v(\cdot), \pi(\cdot)$ be such that, for some $\hat{\theta} \in\left[\hat{\theta}(\rho), p_{M}\right), \pi(\theta)=1$ for all $\theta \geq \hat{\theta}$. If

$$
e^{-\rho v(\hat{\theta})} A e^{-B \hat{\theta}} \frac{1-e^{-(\rho+B)(1-\hat{\theta})}}{\rho+B}=\lambda \frac{A}{B}\left(e^{-B \hat{\theta}}(1-B \hat{\theta})-e^{-B}\right),
$$

then $g(\theta) \geq 0$ for all $\theta \in[\hat{\theta}, 1]$.

Proof (A.19) implies $g(\hat{\theta})=0$. I claim that, under the assumptions of the lemma, one also has $g^{\prime}(\theta) \geq 0$ at $\theta=\hat{\theta}$. For $\theta \geq \hat{\theta}$, (A.18) yields

$$
g^{\prime}(\theta)=\left[\lambda(2-B \theta)-e^{-\rho(v(\hat{\theta})+\theta-\hat{\theta})}\right] A e^{-B \theta} .
$$

Because $\hat{\theta} \geq \hat{\theta}(\rho)$, Lemma A.1 implies that $H(\hat{\theta})>1$. By (A.9), this implies.

$$
(2-B \hat{\theta}) A e^{-B \hat{\theta}} \frac{1-e^{-(\rho+B)(1-\hat{\theta})}}{\rho+B} \geq \frac{A}{B}\left(e^{-B \hat{\theta}}(1-B \hat{\theta})-e^{-B}\right) .
$$

Upon combining (A.21) with (A.19) and noting that, by (A.5), $\hat{\theta}<p_{M}$ implies $e^{-B \hat{\theta}}(1-B \hat{\theta})-e^{-B}$, one obtains

$$
\lambda(2-B \hat{\theta}) \geq e^{-\rho v(\hat{\theta})} .
$$

By (A.20), therefore, $g^{\prime}(\theta) \geq 0$ at $\theta=\hat{\theta}$.

Turning to the proof of the lemma itself, I proceed by contradiction. If the lemma is false, there exists $\bar{\theta} \in(\hat{\theta}, 1]$ such that $g(\bar{\theta})<0$. Because $g(\cdot)$ is continuous, there must exist $\theta_{1} \in[\hat{\theta}, \bar{\theta})$ such that $g\left(\theta_{1}\right)=0$ and $g(\theta)<0$ for all $\theta \in\left(\theta_{1}, \bar{\theta}\right]$. If $\theta_{1}>\hat{\theta}$, 
then, because $g(\hat{\theta})=g\left(\theta_{1}\right)=0$ and $g(\theta)<0$ for $\theta \in\left(\theta_{1}, \bar{\theta}\right]$, there must exist $\theta_{2} \in\left(\hat{\theta}, \theta_{1}\right]$ such that $g(\cdot)$ has a local maximum at $\theta_{2}$. Then

$$
g^{\prime}\left(\theta_{2}\right)=0 \text { and } g^{\prime \prime}\left(\theta_{2}\right) \leq 0 .
$$

If $\theta_{1}=\hat{\theta}$, then, because $g^{\prime}(\hat{\theta}) \geq 0$ and $g(\theta)<0$ for $\theta \in\left(\theta_{1}, \bar{\theta}\right]$, one must have $g^{\prime}(\hat{\theta})=0$ and $g^{\prime \prime}(\hat{\theta}) \leq 0$ so that, by setting $\theta_{2}=\hat{\theta}$, one again obtains (A.23).

By inspection of (A.18), one also has $g(1)=\lambda A e^{-B}>0=g\left(\theta_{1}\right)$. Because $g(\cdot)$ is decreasing at $\theta_{1}$, it follows that there exists $\theta_{3} \in\left(\theta_{1}, 1\right)$ such that $g(\cdot)$ has a minimum at $\theta_{3}$. Then

$$
g^{\prime}\left(\theta_{3}\right)=0 \text { and } g^{\prime \prime}\left(\theta_{3}\right) \leq 0
$$

I claim that (A.23) and (A.24) imply $\theta_{2} \geq \theta_{3}$. From (A.20), one computes

$$
g^{\prime \prime}(\theta)=-B g^{\prime}(\theta)-\left[\lambda B-\rho e^{-\rho(v(\hat{\theta})+\theta-\hat{\theta})}\right] A e^{-B \theta} .
$$

Thus, (A.23) and (A.24) imply

$$
\left[\lambda B-\rho e^{-\rho\left(v(\hat{\theta})+\theta_{2}-\hat{\theta}\right)}\right] \geq\left[\lambda B-\rho e^{-\rho\left(v(\hat{\theta})+\theta_{3}-\hat{\theta}\right)}\right],
$$

which implies $\theta_{2} \geq \theta_{3}$. Yet, by construction, we should have $\theta_{2} \leq \theta_{1}<\theta_{3}$. The assumption that $g(\overline{\bar{\theta}})<0$ for some $\bar{\theta} \in(\hat{\theta}, 1]$ has thus led to a contradiction and must be false.

Proof of Proposition 5.1 I will show that, for $\hat{\theta}(\rho)$ given by Lemma A.1, the admission rule (5.1), (5.2) and the associated indirect utility function $v(\cdot)$ solve the relaxed utilitarian problem. Because the admission rule is nondecreasing, it follows that $\pi(\cdot)$ and $v(\cdot)$ also solve the reduced utilitarian problem. To prove that $\pi(\cdot)$ and $v(\cdot)$ solve the relaxed utilitarian problem, I verify the conditions of Proposition 3.5.

If (5.3) holds, Lemma A. 1 yields $\hat{\theta}(\rho)>0$. The argument in the proof of Lemma A.1 also shows that $H^{\prime}(\hat{\theta}(\rho))>0$. By (A.10), this implies $[\rho(2-B \hat{\theta}(\rho))-B]>0$, hence

$$
0<\frac{B}{\rho(2-B \theta)}<1
$$

for all $\theta \leq \hat{\theta}(\rho)$. (A.27) shows that (5.1) indeed defines an admission probability.

Given the admission rule (5.1), (5.2), calculation of the integral in (3.8), with $Q=1$, yields

$$
v(\theta)=v(\hat{\theta}(\rho))-\frac{1}{\rho} \ln \frac{2-B \theta}{2-B \hat{\theta}(\rho)} \text { if } \theta<\hat{\theta}(\rho)
$$

and

$$
v(\theta)=v(\hat{\theta}(\rho))+\theta-\hat{\theta}(\rho) \text { if } \theta \geq \hat{\theta}(\rho) .
$$


The optimality conditions (3.18) and (3.19) are satisfied by choosing $\lambda$ and $\varphi(\cdot)$ so that

$$
\begin{aligned}
\varphi(\theta)= & \int_{\theta}^{1} e^{-\rho(v(\hat{\theta}(\rho))+\eta-\hat{\theta}(\rho))} A e^{-B \eta} \mathrm{d} \eta-\lambda \frac{A}{B}\left(e^{-B \theta}-e^{-B}\right) \quad \text { if } \theta \geq \hat{\theta}(\rho), \\
\varphi(\theta)= & \int_{\theta}^{\hat{\theta}} \frac{2-B \eta}{2-B \hat{\theta}(\rho)} e^{-\rho v(\hat{\theta}(\rho))} A e^{-B \eta} \mathrm{d} \eta+\int_{\hat{\theta}}^{1} e^{-\rho(v(\hat{\theta}(\rho))+\eta-\hat{\theta}(\rho))} A e^{-B \eta} \mathrm{d} \eta \\
& -\lambda \frac{A}{B}\left(e^{-B \theta}-e^{-B}\right) \text { if } \theta<\hat{\theta}(\rho),
\end{aligned}
$$

and

$$
\lambda=\left[\int_{0}^{\hat{\theta}(\rho)} \frac{2-B \eta}{2-B \hat{\theta}(\rho)} e^{-\rho v(\hat{\theta}(\rho))} A e^{-B \eta} \mathrm{d} \eta+\int_{\hat{\theta}}^{1} e^{-\rho(v(\hat{\theta}(\rho))+\eta-\hat{\theta}(\rho))} A e^{-B \eta} \mathrm{d} \eta\right] .
$$

From (A.31) and (A.32), one obviously has $g(0)=\varphi(0)=0$. From (A.31), one also obtains

$$
\begin{aligned}
g^{\prime}(\theta) & =\lambda\left(f(\theta)+\theta f^{\prime}(\theta)\right)+\varphi^{\prime}(\theta) \\
& =(2-B \theta)\left[\lambda-\frac{1}{2-B \hat{\theta}(\rho)} e^{-\rho v(\hat{\theta}(\rho))}\right] A e^{-B \theta}
\end{aligned}
$$

for $\theta<\hat{\theta}(\rho)$. Because $H(\hat{\theta}(\rho))=1$, (A.32) simplifies to

$$
\lambda=\frac{1}{2-B \hat{\theta}(\rho)} e^{-\rho v(\hat{\theta}(\rho))},
$$

so that (A.33) implies $g^{\prime}(\theta)=0$ for all $\theta \leq \hat{\theta}(\rho)$. Since $g(0)=0$, it follows that $g(\theta)=0$ for all $\theta \leq \hat{\theta}(\rho)$. By Lemma A.2, moreover, $g(\hat{\theta}(\rho))=0$ implies that $g(\theta) \geq 0$ for all $\theta \geq \hat{\theta}(\rho)$. The optimality condition (3.17) is thus also satisfied for all $\theta$. This concludes the proof that, if (5.3) holds, then for $\hat{\theta}(\rho)$ given by Lemma A.1, the admission rule (5.1), (5.2) provides a solution to the relaxed utilitarian problem.

If (5.3) fails to hold, Lemma A.1 yields $\hat{\theta}(\rho)=0$. In this case, (5.2) implies $\pi(\theta)=1$ for all $\theta \in(0,1]$, and (4.2) takes the form (A.18) for all $\theta$. If $\lambda$ is chosen to satisfy (3.19), i.e., if

$$
\lambda=\int_{0}^{1} e^{-\rho(v(0)+\theta)} A e^{-B \theta} \mathrm{d} \theta=e^{-\rho v(0)} A \frac{1-e^{-(\rho+B)}}{\rho+B},
$$


equation (A.19) is satisfied for $\hat{\theta}=0$. By Lemma A.2, one then has $g(\theta) \geq 0$ for all $\theta$, and the optimality condition (3.17) is satisfied. Thus, if (5.3) fails to hold, the admission rule (5.1), (5.2) with $\hat{\theta}(\rho)=0$ provides a solution to the relaxed utilitarian problem.

Proof of Proposition 5.2 I will first show that the solution to the relaxed utilitarian problem must satisfy (5.10) and (5.11) for an appropriate choice of $\hat{\theta}$. Because this result also implies that the admission rule $\pi(\cdot)$ is nondecreasing, the solution to the reduced utilitarian problem coincides with the solution to the relaxed utilitarian problem and therefore also satisfies (5.10) and (5.11) for an appropriate $\hat{\theta}$.

Suppose that $\pi(\cdot)$ and $v(\cdot)$ solve the relaxed utilitarian problem. By Proposition 3.5, there exist $\lambda>0$ and $\varphi(\cdot)$ such that conditions (3.15)-(3.17) hold. As before, $\lambda$ and $\varphi(\cdot)$ are determined by (3.15) and (3.16), or, equivalently, (3.19) and (3.18), so that the key to the analysis is provided by condition (3.17). The maximization in (3.17) is driven by the expression $g(\theta)=\lambda \theta f(\theta)+\varphi(\theta)$. Taking derivatives and using (3.16), one obtains

$$
\begin{aligned}
g^{\prime}(\theta) & =\lambda\left(f(\theta)+\theta f^{\prime}(\theta)\right)+\varphi^{\prime}(\theta) \\
& =\left[\lambda\left(2+\frac{\theta f^{\prime}(\theta)}{f(\theta)}\right)-W^{\prime}(v(\theta))\right] f(\theta) .
\end{aligned}
$$

Because $v(\theta)$ is nondecreasing and $W$ is strictly concave, the term $-W^{\prime}(v(\theta))$ is nondecreasing in $\theta$. Because the elasticity $\frac{\theta f^{\prime}(\theta)}{f(\theta)}$ is also nondecreasing, it follows that the term in square brackets is nondecreasing in $\theta$. Thus, if $g^{\prime}(\theta)>0$ for some $\theta$, one infers that $g^{\prime}\left(\theta^{\prime}\right)>0$ for all $\theta^{\prime}>\theta$.

The term in brackets is actually strictly increasing at any $\theta$ at which $v(\cdot)$ is strictly increasing, i.e., at any $\theta$ with $\pi(\theta)>0$. Thus, if $g^{\prime}(\theta)=0$ for some $\theta$, then, for $\theta^{\prime}>\theta$, one infers that $g^{\prime}\left(\theta^{\prime}\right) \geq 0$, and that $g^{\prime}\left(\theta^{\prime}\right)=0$ only if $\pi\left(\theta^{\prime \prime}\right)=g^{\prime}\left(\theta^{\prime \prime}\right)=0$ for all $\theta^{\prime \prime} \in\left(\theta, \theta^{\prime}\right)$.

These observations imply that, if $g^{\prime}(0)>0$, then one has $g^{\prime}(\theta)>0$ for all $\theta$. This implies that $g(\theta)>g(0)=0$ for all $\theta>0$. The optimality condition (3.17) then requires that $\pi(\theta)=1$ for all $\theta \in(0,1]$. The admission rule satisfies (5.10) and (5.11) with $\hat{\theta}=0$.

Alternatively, if $g^{\prime}(0)=0$, one may let $\bar{\theta}=\sup \left\{\theta \mid g^{\prime}(\theta)=0\right\}$. If $\bar{\theta}=0$, the same argument as before requires that $g(\theta)>g(0)=0$ and therefore $\pi(\theta)=1$ for all $\theta \in(0,1]$. If $\bar{\theta}>0$, one must have $\pi(\theta)=0$ and $g^{\prime}(\theta)=0$ for all $\theta \in(0, \bar{\theta})$. It follows that $g(\bar{\theta})=g(0)=0$. Moreover, by the same argument as before, $g(\theta)>g(\bar{\theta})=0$ and therefore $\pi(\theta)=1$ for all $\theta \in(\bar{\theta}, 1]$. In either case, if $\bar{\theta}=0$ and if $\bar{\theta}>0$, the admission rule satisfies (5.10) and (5.11) with $\hat{\theta}=\bar{\theta}$.

Finally, if $g^{\prime}(0)<0$, the function $g(\cdot)$ achieves a minimum at some $\check{\theta}>0$. Since $g(0)=0$, one has $g(\check{\theta})<0$, as well as $g^{\prime}(\check{\theta})=0$. Since $g(1)=\lambda f(1)>0$, one has $\check{\theta}<1$. Between $\theta=\check{\theta}$ and $\theta=1, g(\theta)$ rises monotonically from $g(\check{\theta})$ to $g(1)$. There is then a unique $\hat{\theta} \in(\check{\theta}, 1)$ so that $g(\hat{\theta})=0$, and $g(\theta)<0$ for $\theta \in(0, \hat{\theta}), g(\theta)>0$ for $\theta \in(\hat{\theta}, 1]$. In this case, (3.17) requires that $\pi(\theta)=0$ for $\theta \in(0, \hat{\theta})$ and $\pi(\theta)=1$ for $\theta \in(\hat{\theta}, 1]$. The admission rule satisfies $(5.10)$ and (5.11) for this critical $\hat{\theta}$. 
Proposition 4.3 also implies $\hat{\theta} \leq p_{M}$. By (4.2), moreover, $g(\hat{\theta})=0$ implies $\hat{\theta} f(\hat{\theta})<1-F(\hat{\theta})$, so that one must have $\hat{\theta} \neq p_{M}$, and hence $\hat{\theta}<p_{M}$.

The preceding arguments have shown that $\hat{\theta}=0$ if $g^{\prime}(\theta)>0$ for $\theta$ arbitrarily close to zero and $\hat{\theta}>0$ if $g^{\prime}(\theta) \leq 0$ for $\theta$ arbitrarily close to zero. Thus $\hat{\theta}>0$ implies $g^{\prime}(0) \leq 0$. By (A.35) and (3.19), one must then have

$$
W^{\prime}(v(0)) \geq 2 \lambda=2 \int_{0}^{1} W^{\prime}(v(\theta)) f(\theta) \mathrm{d} \theta .
$$

Since $v(\theta)=v(0)+\max (\theta-\hat{\theta}, 0)<v(0)+\theta$ for all $\theta$ and $W$ is strictly concave, (5.12) follows.

Similarly, $\hat{\theta}=0$ implies $g^{\prime}(0) \geq 0$. By (A.35) and (3.19), one must then have

$$
W^{\prime}(v(0)) \leq 2 \lambda=2 \int_{0}^{1} W^{\prime}(v(\theta)) f(\theta) \mathrm{d} \theta
$$

Since $\hat{\theta}=0$ implies $v(\theta)=v(0)+\theta$ for all $\theta$, (A.37) implies that (5.12) is violated. Conversely, (5.12) implies that $\hat{\theta}>0$.

Proof of Proposition 6.1 I will show that the Dupuit admission rule and the indirect utility function that the Dupuit admission rule induces solve the relaxed utilitarian problem with participation constraints if inequality aversion is sufficiently small. For this purpose, I verify the optimality conditions of Proposition 3.5, with $\varphi(0)=0$ in (3.15) replaced by the new transversality condition $\varphi(0) \leq 0$.

If $\lambda$ is specified so that $g(\bar{\theta})=0$, i.e., if

$$
\lambda=\frac{\int_{\bar{\theta}}^{1} W^{\prime}(Y+\eta-\bar{\theta}) f(\eta) d \eta}{1-F(\bar{\theta})-\bar{\theta} f(\bar{\theta})},
$$

and $\varphi(\cdot)$ is given by (3.18), then the transversality condition $\varphi(1)=0$ and (3.16) hold automatically. As in the proof of Proposition 4.1, $\rho_{W}(v) \leq A$ for all $v$ implies

$$
W^{\prime}(Y+\eta) \geq W^{\prime}(Y) e^{-A \eta}
$$

for all $\eta$. By (A.38), therefore,

$$
\lambda \geq \frac{\int_{\bar{\theta}}^{1} W^{\prime}(Y) e^{-A} f(\theta) \mathrm{d} \theta}{1-F(\bar{\theta})-\bar{\theta} f(\bar{\theta})} \geq e^{-A} \frac{1-F(\bar{\theta})}{1-F(\bar{\theta})-\bar{\theta} f(\bar{\theta})} W^{\prime}(Y) .
$$

If $A$ is sufficiently close to zero so that

$$
e^{-A} \frac{1-F(\bar{\theta})}{1-F(\bar{\theta})-\bar{\theta} f(\bar{\theta})}>1
$$


the right-hand side of (A.40) is greater that $W^{\prime}(Y)$. By the concavity of $W, W^{\prime}(Y)$ in turn is greater than $\int_{0}^{1} W^{\prime}(Y+\max (\theta-\bar{\theta}, 0)) f(\theta) \mathrm{d} \theta$. By (3.18), one then has $\varphi(0)<0$, and the transversality condition for $v(0)$ is satisfied.

To complete the proof of the proposition, I verify that

$$
g(\theta) \lesseqgtr 0 \quad \text { as } \theta \lesseqgtr \bar{\theta}
$$

so that the optimality condition (3.17) is also satisfied. By inspection of (4.2), (A.41) is equivalent to the requirement that, for $\theta$

$$
\frac{\int_{\theta}^{1} W^{\prime}(Y+\max (\eta-\bar{\theta}, 0)) f(\eta) \mathrm{d} \eta}{1-F(\theta)}-\lambda \frac{1-F(\theta)-\theta f(\theta)}{1-F(\theta)} \lesseqgtr \text { as } \theta \lesseqgtr \bar{\theta} \text {. }
$$

To prove (A.42), I first note that, by the definition of $\lambda$, the two sides of (A.42) are equal if $\theta=\bar{\theta}$. I also observe that, for $\theta=p_{M}$, one has $1-F(\theta)-\theta f(\theta)=0$. By the assumption that $\frac{\theta f(\theta)}{1-F(\theta)}$ is increasing in $\theta$, it follows that, for all $\theta \geq p_{M}, 1-F(\theta)-$ $\theta f(\theta) \leq 0$ so that the left-hand side of (A.42) is positive, as required by (A.42).

To complete the proof of (A.42), I show that the derivative of the left-hand side of (A.42) with respect to $\theta$ is positive for all $\theta<p_{M}$, if inequality aversion is sufficiently small. The derivative of the second term on the left-hand side is bounded below by $\lambda B$, where $B>0$ is the given lower bound on $\frac{\mathrm{d}}{\mathrm{d} \theta} \frac{\theta f(\theta)}{1-F(\theta)}$. As for the first term, one computes

$$
\begin{aligned}
& \frac{\mathrm{d}}{\mathrm{d} \theta} \frac{\int_{\theta}^{1} W^{\prime}(Y+\max (\eta-\bar{\theta}, 0)) f(\eta) \mathrm{d} \eta}{1-F(\theta)} \\
& \quad=\frac{f(\theta)}{1-F(\theta)}\left[\frac{\int_{\theta}^{1} W^{\prime}(Y+\max (\eta-\bar{\theta}, 0)) f(\eta) d \eta}{1-F(\theta)}-W^{\prime}(Y+\max (\theta-\bar{\theta}, 0))\right] \\
& \quad \geq \frac{f(\theta)}{1-F(\theta)}\left[W^{\prime}(Y+1)-W^{\prime}(Y)\right] \\
& \quad \geq \frac{f(\theta)}{1-F(\theta)} W^{\prime}(Y)\left(e^{-A}-1\right),
\end{aligned}
$$

where $A$ is again the upper bound on the inequality aversion $\rho_{W}(v)$. If $A$ is sufficiently close to zero, one obviously has

$$
\frac{f(\theta)}{1-F(\theta)} W^{\prime}(Y)\left(e^{-A}-1\right)+\lambda B>0
$$

for all $\theta<p_{M}$, so that the left-hand side of (A.42) is indeed increasing in $\theta$ on the interval $\left[0, p_{M}\right)$. The validity of (A.42) and (A.41) follows immediately.

Proof of Proposition 6.2 The threshold $\hat{\theta}(\rho)$ is again given by Lemma A.1. There are two cases to be considered: 
Case $1 \hat{\theta}(\rho) \leq \bar{\theta}$. I claim that, in this case, the Dupuit admission rule solves the relaxed utilitarian problem with participation constraints. To prove this claim, I show that this rule satisfies the optimality conditions of the relaxed utilitarian problem with participation constraints. For $\theta \geq \bar{\theta}$, under the Dupuit admission rule, $g(\theta)$ is given by (A.18). If $\lambda$ is such that $g(\bar{\theta})=0$, then, by Lemma A.2, one has $g(\theta) \geq 0$ for all $\theta \in[\bar{\theta}, 1]$. The optimality condition (3.17) is thus satisfied for $\theta \geq \bar{\theta}$.

For $\theta<\bar{\theta}$, under the Dupuit rule, (3.18) yields

$$
\varphi(\theta)=\int_{\theta}^{\bar{\theta}} e^{-\rho Y} A e^{-B \eta} \mathrm{d} \eta+\int_{\hat{\theta}}^{1} e^{-\rho(Y+\eta-\bar{\theta})} A e^{-B \eta} \mathrm{d} \eta-\lambda \frac{A}{B}\left(e^{-B \theta}-e^{-B}\right),
$$

hence

$$
\begin{aligned}
g^{\prime}(\theta) & =\lambda(1-B \theta) A e^{-B \theta}+\varphi^{\prime}(\theta) \\
& =\left[\lambda(2-B \theta)-e^{-\rho Y}\right] A e^{-B \theta} .
\end{aligned}
$$

I claim that

$$
\lambda(2-B \theta) \geq e^{-\rho Y}
$$

hence $g^{\prime}(\theta) \geq 0$ for all $\theta \leq \bar{\theta}$. Because $g(\bar{\theta})=0$, this implies $g(\theta) \leq 0$ for all $\theta \leq \bar{\theta}$, so that the optimality condition (3.17) is satisfied for $\theta \leq \bar{\theta}$ as well as $\theta \geq \bar{\theta}$. Since $g(0)=\varphi(0)$, the transversality condition for $v(0)$ is then satisfied as well.

To prove (A.44), I note that, by Lemma A. $1, \bar{\theta} \geq \hat{\theta}(\rho)$ implies $H(\bar{\theta}) \geq 1$. By (A.9), it follows that

$$
(2-B \bar{\theta}) A e^{-B \bar{\theta}} \frac{1-e^{-(\rho+B)(1-\bar{\theta})}}{\rho+B} \geq \frac{A}{B}\left[e^{-B \bar{\theta}}(1-B \bar{\theta})-e^{-B}\right] .
$$

Because $\lambda$ has been chosen so that $g(\bar{\theta})=\lambda \bar{\theta} A e^{-B \bar{\theta}}+\varphi(\bar{\theta})=0$, one also has

$$
e^{-\rho Y} A e^{-B \bar{\theta}} \frac{1-e^{-(\rho+B)(1-\bar{\theta})}}{\rho+B}=\lambda \frac{A}{B}\left[e^{-B \bar{\theta}}(1-B \bar{\theta})-e^{-B}\right] .
$$

Upon combining (A.45) and (A.46), one finds that $\lambda(2-B \bar{\theta}) \geq e^{-\rho Y}$. Therefore, (A.44) must hold for all $\theta \leq \bar{\theta}$. The Dupuit admission rule thus satisfies the optimality conditions of the relaxed utilitarian problem with participation constraints.

Case $2 \hat{\theta}(\rho)>\bar{\theta}$. If $\rho$ is large enough so that the solution to the reduced utilitarian problem without participation constraints satisfies the participation constraint (6.1) anyway, the claims of the proposition for this case follow directly from Proposition 5.1. Suppose, therefore, that the solution to the reduced utilitarian problem without participation constraints violates (6.1). The participation constraint must then be binding 
in the reduced utilitarian problem with participation constraints, i.e., one must have $v(0)=Y$, and the feasibility constraint is equivalent to (6.4). It is convenient to rewrite this in the form

$$
\bar{K} \leq \int_{0}^{1} \pi(\theta)(\theta f(\theta)-(1-F(\theta))) \mathrm{d} \theta
$$

using repeated integration by parts.

I claim that there is a unique $\theta^{*}(\rho)$ so that the admission rule specified in (6.6)-(6.8) satisfies (A.47) as an equation. To see this, take any $\theta^{*} \in[0, \hat{\theta}(\rho)]$ and consider the admission rule

$$
\begin{aligned}
& \pi\left(\theta \mid \theta^{*}\right)=0 \text { if } \theta \in\left[0, \theta^{*}\right) \\
& \pi\left(\theta \mid \theta^{*}\right)=\frac{B}{\rho(2-B \theta)} \in(0,1) \text { if } \theta \in\left[\theta^{*}, \hat{\theta}(\rho)\right),
\end{aligned}
$$

and

$$
\pi\left(\theta \mid \theta^{*}\right)=1 \quad \text { if } \theta \in(\hat{\theta}(\rho), 1]
$$

For $\theta^{*}=0$, the admission rule $\pi\left(\cdot \mid \theta^{*}\right)$ is the same as in Proposition 5.1 which, by assumption, violates (A.47). For $\theta^{*}=\hat{\theta}(\rho), \pi\left(\cdot \mid \theta^{*}\right)$ provides for admission if and only if people pay the admission fee $\hat{\theta}(\rho)$; under this rule, the right-hand side of (A.47) is equal to $\hat{\theta}(\rho)\left(1-F(\hat{\theta}(\rho))\right.$. Because $\hat{\theta}(\rho)$ lies between $\bar{\theta}$ and $p_{M}$, one has $\hat{\theta}(\rho)(1-F(\hat{\theta}(\rho))>\bar{\theta}(1-F(\bar{\theta}))=\bar{K}$. By the intermediate value theorem, therefore, there exists $\theta^{*}(\rho) \in(0, \hat{\theta}(\rho))$ such that

$$
\int_{0}^{1} \pi\left(\theta \mid \theta^{*}(\rho)\right)(\theta f(\theta)-(1-F(\theta))) \mathrm{d} \theta=\bar{K} .
$$

Using (A.5), one easily verifies that, on the interval $[0, \hat{\theta}(\rho)]$ the map

$$
\theta \rightarrow \int_{0}^{1} \pi\left(\theta \mid \theta^{*}\right)(\theta f(\theta)-(1-F(\theta))) \mathrm{d} \theta
$$

is increasing; the solution $\theta^{*}(\rho)$ to Eq. (A.51) is therefore unique.

To complete the proof, I show that the stipulated admission rule and the associated indirect utility function satisfy the optimality conditions for the relaxed utilitarian problem with participation constraints. The indirect utility function is given as

$$
\begin{aligned}
& v(\theta)=Y \quad \text { if } \theta \in\left[0, \theta^{*}(\rho)\right], \\
& v(\theta)=Y-\frac{1}{\rho} \ln \frac{2-B \theta}{2-B \theta^{*}(\rho)} \quad \text { if } \theta \in\left[\theta^{*}(\rho), \hat{\theta}(\rho)\right],
\end{aligned}
$$


and

$$
v(\theta)=Y-\frac{1}{\rho} \ln \frac{2-B \hat{\theta}(\rho)}{2-B \theta^{*}(\rho)}+\theta-\hat{\theta}(\rho) \text { if } \theta \in[\hat{\theta}(\rho), 1]
$$

To verify the optimality conditions for the relaxed utilitarian problem with participation constraints, I specify $\lambda$ and $\varphi$ so that

$$
\begin{aligned}
\lambda= & \frac{e^{-\rho v(\hat{\theta}(\rho))}}{2-B \hat{\theta}(\rho)}, \\
\varphi(\theta)= & \int_{\theta}^{1} e^{-\rho(v(\hat{\theta}(\rho))+\eta-\hat{\theta}(\rho))} A e^{-B \eta} \mathrm{d} \eta-\lambda \frac{A}{B}\left(e^{-B \theta}-e^{-B}\right) \quad \text { if } \theta \in[\hat{\theta}(\rho), 1] \\
\varphi(\theta)= & \int_{\theta}^{\hat{\theta}(\rho)} \frac{2-B \eta}{2-B \hat{\theta}(\rho)} e^{-\rho v(\hat{\theta}(\rho))} A e^{-B \eta} \mathrm{d} \eta+\int_{\hat{\theta}(\rho)}^{1} e^{-\rho(v(\hat{\theta}(\rho))+\eta-\hat{\theta}(\rho))} A e^{-B \eta} \mathrm{d} \eta \\
& -\lambda \frac{A}{B}\left(e^{-B \theta}-e^{-B}\right) \text { if } \theta \in\left[\theta^{*}(\rho), \hat{\theta}(\rho)\right]
\end{aligned}
$$

and

$$
\begin{aligned}
\varphi(\theta)= & \int_{\theta}^{\theta^{*}(\rho)} e^{-\rho Y} A e^{-B \eta} \mathrm{d} \eta+\int_{\theta^{*}(\rho)}^{\hat{\theta}(\rho)} \frac{2-B \eta}{2-B \hat{\theta}(\rho)} e^{-\rho v(\hat{\theta}(\rho))} A e^{-B \eta} \mathrm{d} \eta \\
& +\int_{\hat{\theta}(\rho)}^{1} e^{-\rho(v(\hat{\theta}(\rho))+\eta-\hat{\theta}(\rho))} A e^{-B \eta} \mathrm{d} \eta-\lambda \frac{A}{B}\left(e^{-B \theta}-e^{-B}\right) \\
& \text { if } \theta \in\left[0, \theta^{*}(\rho)\right] .
\end{aligned}
$$

The transversality condition $\varphi(1)=0$ and the differential equation (3.16) are automatically satisfied. It remains to be shown that

$$
\begin{aligned}
& g(\theta)=\lambda \theta f(\theta)+\varphi(\theta) \leq 0 \quad \text { if } \theta<\theta^{*}(\rho), \\
& g(\theta)=\lambda \theta f(\theta)+\varphi(\theta)=0 \text { if } \theta \in\left(\theta^{*}(\rho), \hat{\theta}(\rho)\right)
\end{aligned}
$$

and

$$
g(\theta)=\lambda \theta f(\theta)+\varphi(\theta) \geq 0 \text { if } \theta>\hat{\theta}(\rho)
$$

Since $g(0)=\varphi(0)$, the transversality condition $\varphi(0) \leq 0$ is automatically implied by (A.59). 
To prove (A.59)-(A.61), I first note that (A.55) and (A.56) imply

$$
\varphi(\hat{\theta}(\rho))=\lambda(2-B \hat{\theta}(\rho)) \int_{\hat{\theta}(\rho)}^{1} e^{-\rho(\theta-\hat{\theta}(\rho))} A e^{-B \theta} \mathrm{d} \theta-\lambda \frac{A}{B}\left(e^{-B \hat{\theta}(\rho)}-e^{-B}\right) .
$$

By (A.9) and the fact that $H(\hat{\theta}(\rho))=1$, it follows that

$$
\varphi(\hat{\theta}(\rho))=-\lambda A \hat{\theta}(\rho) e^{-B \hat{\theta}(\rho)},
$$

and hence that $g(\hat{\theta}(\rho))=\lambda \hat{\theta}(\rho) f(\hat{\theta}(\rho))+\varphi(\hat{\theta}(\rho))=0$. (A.61) then follows from Lemma A.2. For $\theta \in\left(\theta^{*}(\rho), \hat{\theta}(\rho)\right)$, (A.57) yields

$$
g^{\prime}(\theta)=\left[\lambda(2-B \theta)-e^{-\rho v(\hat{\theta}(\rho))} \frac{2-B \theta}{2-B \hat{\theta}(\rho)}\right] A e^{-B \theta},
$$

so that (A.55) implies $g^{\prime}(\theta)=0$ for all $\theta \in\left(\theta^{*}(\rho), \hat{\theta}(\rho)\right)$. Since $g(\hat{\theta}(\rho))=0$, (A.60) follows. Finally, for $\theta<\theta^{*}(\rho)$, (A.58) and (A.55) yield

$g^{\prime}(\theta)=\left[\lambda(2-B \theta)-e^{-\rho Y}\right] A e^{-B \theta}=(2-B \theta)\left[\frac{e^{-\rho v(\hat{\theta}(\rho))}}{2-B \hat{\theta}(\rho)}-\frac{e^{-\rho Y}}{2-B \theta}\right] A e^{-B \theta}$.

By (A.53), therefore,

$$
\begin{aligned}
g^{\prime}(\theta) & =(2-B \theta)\left[\frac{e^{-\rho v\left(\theta^{*}(\rho)\right)}}{2-B \theta^{*}(\rho)}-\frac{e^{-\rho Y}}{2-B \theta}\right] A e^{-B \theta} \\
& =e^{-\rho Y}\left[\frac{2-B \theta}{2-B \theta^{*}(\rho)}-1\right] A e^{-B \theta}>0
\end{aligned}
$$

for $\theta<\theta^{*}(\rho)$. Since $g\left(\theta^{*}(\rho)\right)=0$, (A.59) follows. This completes the proof that, if $\bar{\theta}<\hat{\theta}(\rho)$, the specified admission rule satisfies the optimality conditions of the relaxed utilitarian problem with participation constraints.

The proof of Proposition 6.3 is identical to the proof of Proposition 5.2 and is left to the reader.

Open Access This article is distributed under the terms of the Creative Commons Attribution Noncommercial License which permits any noncommercial use, distribution, and reproduction in any medium, provided the original author(s) and source are credited.

\section{References}

Atkinson, A.B.: How progressive should income tax be? In: Parkin, M., Nobay, R. (eds.) Essays on Modern Economics. London: Longman (1973) 
Brito, D.L., Hamilton, J.H., Slutsky, S.M., Stiglitz, J.E.: Randomization in optimal income tax schedules. J Public Econ 56, 189-223 (1995)

Clarke, F.H.: Optimization and Nonsmooth Analysis. New York: Wiley (1983)

Dupuit, J.: De la mesure de l'utilité des travaux publics. Annales des ponts et chaussées, $2^{e}$ série. Mémoires et Documents 116(VIII), 332-375 (1844)

Feldman, M., Gilles, C.: An expository note on individual risk without aggregate uncertainty. J Econ Theory 35, 26-32 (1985)

Fudenberg, D., Tirole, J.: Game Theory. Cambridge: MIT Press (1991)

Guesnerie, R., Laffont, J.-J.: A complete solution to a class of principal-agent problems with an application to the control of a self-managed firm. J Public Econ 25, 329-369 (1984)

Hellwig, M.F.: A utilitarian approach to the provision and pricing of excludable public goods. J Public Econ 89, 1981-2003 (2005)

Hellwig, M.F.: The undesirability of randomized income taxation under decreasing risk aversion. J Public Econ 91, 791-816 (2007)

Hellwig, M.F.: A contribution to the theory of optimal utilitarian income taxation. J Public Econ 91, 14491477 (2007)

Hellwig, M.F.: A maximum principle for control problems with monotonicity constraints. Max Planck Institute for Research on Collective Goods, Bonn, Germany. http://www.coll.mpg.de/pdf_dat/ 2008_04online.pdf (2008)

Hellwig, M.F.: Utilitarian mechanism design for an excludable public good. Max Planck Institute for Research on Collective Goods, Bonn, Germany. http://www.coll.mpg.de/pdf_dat/2009_12online.pdf (2009)

Judd, K.L.: The law of large numbers with a continuum of IID random variables. J Econ Theory 35, 19$25(1985)$

Khan, M.A., Sun, Y.N.: Weak measurability and charactarizations of risk. Econ Theory 13, 541-560 (1999)

Manelli, A., Vincent, D.: Bundling as an optimal selling mechanism for a multiple-good monopolist. J Econ Theory 127, 1-35 (2006)

Mangasarian, O.L.: Sufficient conditions for the optimal control of nonlinear systems. SIAM J Control 4, 139-152 (1966)

Mirrlees, J.M.: An exploration in the theory of optimum income taxation. Rev Econ Stud 38, 175-208 (1971)

Mirrlees, J.M.: Optimal tax theory: a synthesis. J Public Econ 6, 327-358 (1976)

Norman, P.: Efficient mechanisms for public goods with use exclusion. Rev Econ Stud 71, 1163-1188 (2004)

Podczeck, K.: On existence of rich Fubini extensions. Econ Theory (2009). doi:10.1007/s00199-009-0458-9

Roberts, K.W.S.: Voting over income tax schedules. J Public Econ 8, 329-340 (1977)

Schmitz, P.W.: Monopolistic provision of excludable public goods under private information. Public Financ 52, 89-101 (1997)

Stiglitz, J.E.: Self-selection and Pareto efficient taxation. J Public Econ 17, 213-240 (1982)

Sun, Y.N.: The exact law of large numbers via Fubini extension and characterization of insurable risks. J Econ Theory 126, 31-69 (2006)

Sun, Y.N., Zhang, Y.: Individual risk and Lebesgue extension without aggregate uncertainty. J Econ Theory 144, 432-442 (2009) 